

\section{DISCLAIMER}

This report was prepared as an account of work sponsored by an agency of the United States Government. Neither the United States Government nor any agency Thereof, nor any of their employees, makes any warranty, express or implied, or assumes any legal liability or responsibility for the accuracy, completeness, or usefulness of any information, apparatus, product, or process disclosed, or represents that its use would not infringe privately owned rights. Reference herein to any specific commercial product, process, or service by trade name, trademark, manufacturer, or otherwise does not necessarily constitute or imply its endorsement, recommendation, or favoring by the United States Government or any agency thereof. The views and opinions of authors expressed herein do not necessarily state or reflect those of the United States Government or any agency thereof. 


\section{DISCLAIMER}

Portions of this document may be illegible in electronic image products. Images are produced from the best available original document. 


\section{DISCLAIMER}

This report was prepared as an account of work sponsored by an agency of the United States Covernment. Neither the United States Government nor any agency thereof, nor any of their employees, makes any warranty, express or implied, or assumes any legal liability or responsibility for the accuracy, completeness, or usefulness of any information, apparatus, product, or process disclosed, or represents that its use would not infringe privately owned rights. Reference herein to any specific commercial product, process, or service by trade name, trademark, manufacturer, or otherwise, does not necessarily constitute or imply its endorsement, recommendation, or favoring by the United States Government or any agency thereof. The views and opinions of authors expressed herein do not necessarily state or reflect those of the United States Government or any agency thereof.

Printed in the United States of America

Available from

National Technical Information Service

U.S. Department of Commerce

5285 Port Royal Road

Springfield, VA 22161

NTIS price codes $\mathrm{AO} 3$

Printed Copy:

Microfiche copy: A01 
MLM-3348

UC.4

\title{
Surface Characterization of an Energetic Material, Pentaerythritoltetranitrate (PETN), Having a Thin Coating Achieved Through a Starved Addition Microencapsulation Technique
}

\author{
Cynthia M. Worley
}

Issued: May 7, 1986

\section{DISCLAIMER}

\begin{abstract}
This repont was prepared as an account of work sponsored by an agency of the United States Government. Neither the United States Government nor any agency thereof, nor any of their employees, makes any warranty, express or implied, or assumes any legal liability or responsibility for the accuracy, completeness, or usefulness of any information, apparatus, product, or process disclosed, or represents that its use would not infringe privately owned rights. Reference herein to any specific commercial product, process, or service by trade name, trademark, manufacturer, or otherwise does not necessarily constitute or imply its endorsement, recommendation, or favoring by the United States Government or any agency thereof. The views and opinions of authors expressed herein do not necessarily state or reflect those of the United States Government or any agency thereof.
\end{abstract}

\section{MOUND}

Miamisburg, Onio 45342

operated by

\section{MONSANTO RESEARCH CORPORATION}

a subsidiary of Monsanto Company

for the

U. S. DEPARTMENT OF ENERGY

Contract NO DE-ACO4-76-DPOOOS3 


\section{Contents}

page

ABSTRACT ............................ . . 3

1. INTRODUCTION . . . . . . . . . . . . . . . . . . 3

2. EXPERTMENTAL . . . . . . . . . . . . . . . . . . . . 4 4

2.1. Application of a Coating Using a starved Addition

Enhanced Microencapsulation Technique . . . . . . . 4

2.2. Surface Analysis . . . . . . . . . . . . . . . . 4

2.2.1. Optical Microscopy . . . . . . . . . . . 5

2.2.2. Scanning Electron Microscopy (SEM) . . . . . 5

2.2.3. X-Ray Photoelectron Spectroscopy (XPS) . . . 6

2.2.4. Ion Scattering Spectroscopy (ISS) . . . . . 6

2.3. Particle Size - Coulter counter . . . . . . . . . 6

2.4. Contact Angle Measurement . . . . . . . . . . . 7

3. RESULTS AND DISCUSSION . . . . . . . . . . . . . . . 7

3.1. Bonding in Microencapsulation Enhanced with

Starved Addition................ 16

3.2. Surface Wettability ................ . . 17

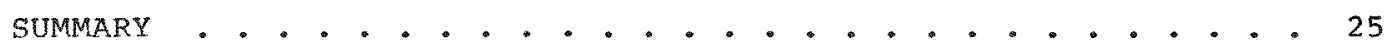

REFERENCES . . . . . . . . . . . . . . . . . . . . . . 26

APPENDIX A . . . . . . . . . . . . . . . . . . 27

APPENDIX B....................... 32

APPENDIX C . . . . . . . . . . . . . . . . . . 35

DISTRIBUTION ... . . . . . . . . . . . . . . . . 47 


\begin{abstract}
The objective of this research was to: (1) determine the nature of a thin coating on an explosive material which was applied using a starved addition microencapsulation technique, (2) understand the coating/crystal bond, and (3) investigate the wettability/adhesion of plastic/solvent combinations using the coating process. The coating used in this work was a Firestone Plastic Company copolymer (FPC-461) of vinylchloride/trifluorochloroethylene in a $1.5 / 1.0$ weight ratio. The energetic explosive examined was pentaerythritoltetranitrate (PETN). The coating process used was starved addition followed by a solvent evaporation technique.
\end{abstract}

Surface analytical studies, completed for characterization of the coating process, show (1) evidence that the polymer coating is present, but not continuous, over the surface of PETN; (2) the average thickness of the polymer coating is between $16-32 \AA$ and greater than $44 \AA$, respectively, for 0.5 and 20 wt 8 coated PETN; (3) no changes in surface chemistry of the polymer or the explosive material following microencapsulation; and (4) the presence of explosive material on the surface of 0.5 wt FPC-461 coated explosives.

\section{Introduction}

This work involved characterization of very thin plastic coatings. The coatings are on fine, organic, solid, crystalline, explosive powders. They were applied using a microencapsulation type processing technique, previously developed at Monsanto Research Corporation, Mound, which incorporates a starved addition of polymer in solution to a suspension of the powder crystals. The coating is distinguished by the use of as little as 0.5 wt $\&$ (relative to the weight of the powder particle) plastic.

Microencapsulation techniques generally are used to apply plastic coatings around solid particles and liquid droplets, but normally require 20 wt $\delta$ plastic. The purpose of the very thin coatings, evaluated herein, was to provide an adhesive to bind needle-like or irregularly shaped particles together into spherical prills which have improved pourability. This is in contrast to many microencapsulation applications where it is desirable to completely surround, or encapsulate, a core material.

The goals of this research were (1) to determine the nature of the very thin coatings which are applied to explosive powders through a starved addition microencapsulation process technique; (2) to understand the nature of the coating/ crystal powder interface; and (3) to investigate the wetting abilities of the solvent/plastic combinations used in the coating process.

The coating studied was a copolymer of vinylchloride/trifluorochloroethylene (1.5/1.0 molar ratio) supplied by Firestone Plastic Company as FPC-461. 
The energetic (explosive) powder material studied was pentaerythritoltetranitrate (PETN).

X-ray photoelectron spectroscopy (XPS) and ion scattering spectroscopy (ISS) results indicate that the explosive powder particles are being partially coated as desired. Since no change in the surface chemistry of the explosive or polymer could be noted in the XPS analysis, the bonding between PETN and FPC-461 is likely mechanical.

The contact angle measurements indicate that low surface tension solvents should be used in the microencapsulation enhanced with starved adition process. Low contact angles and, therefore, sufficiently high wettability are achieved using this type of solvent.

Background studies were completed for (1) the history, description, and process parameters of microencapsulation; application of a coating by a starved addition enhanced microencapsulation technique; and (3) surface analytical techniques used for the characterization work. This information can be found in Appendices A, B, and C, respectively.

\section{Experimental}

\subsection{Application of a Coating Using a Starved Addition Enhanced \\ Microencapsulation Technique}

The PETN powder material used in this work was purchased from dupont. The material was then processed at Monsanto Research Corporation (Mound). The PETN powder was precipitated from an acetone, ethanol, and water solution by a standard addition method. standard addition implies that the nonsolvent was added to the solvent. When this process was completed, the PETN powder could be coated using the microencapsulation enhanced with starved addition technique. [1]

One hundred gram batches of 0.5 and 20 wt $\%$ coated PETN were made for experimental use. In order to synthesize the hatch of 0.5 wt 8 coated PETN, $0.5 \mathrm{~g}$ of FPC-461 (coating) was added to $500 \mathrm{mI}$ of methylene chloride. FPC-461 was dissolved in the methylene chloride by gentle (Iow) heating and stirring. Then, $99.5 \mathrm{~g}$ of PETN was added to $600 \mathrm{~g}$ of ethanol and $4000 \mathrm{~g}$ of water. The PETN, ethanol, and water solution was stirred as the FPC-461/methylene chloride solution was added arop by arop through an addition funne1. When the FPC-461/methylene chloride addition was completed, the solution was held at $45^{\circ} \mathrm{C}$ for $30 \mathrm{~min}$ to remove the solvent. The product was then air aried using a vacuum, and then was oven dried to obtain the easier handling particles.

The same process used for the 0.5 wt \& coated PETN was used to prepare the 20 wt \& batch. The only difference was the amounts of materials used; that is, 20 व of FPC-461, $1000 \mathrm{mr}$ of methylene chloride, and $80 \mathrm{~g}$ of PETN.

\subsection{Surface Analysis}

There are many different techniques that can be used for the characterization of a surface. Because of the nature of the sample materials used in this work, techniques that were virtually nondestructive were necessary. The techniques chosen for surface characterization of the energetic materials were optical microscopy, scanning electron microscopy (SEM), SEM backscattering, $x$-ray photoelectron spectroscopy (XPS), and ion 
scattering spectroscopy (ISS). (Each technique is briefly described in Appendix $C$. Brief sections on particle size and contact angle measurement also appear in Appendix C.)

The goal was to determine the distribution and the nature of bonding of the coated PETN material obtained by this technique of microencapsulation enhanced with starved addition. Table 1 summarizes the expected results and sampling depths of the surface analytical techniques used. The following sections discuss the experimental equipment used for each of the surface characterization technigues.

\subsubsection{OPTICAL MICROSCOPY}

A Wild M5A Heerbrugg stereo Microscope with a schott KL1500 cold light source was used for viewing the FPC-461 and the coated and uncoated PETN samples. A Polaroid $\mathrm{sx}-70$ camera was attached to the microscope. Photographs were taken at $15 \mathrm{X}$ magnification.

\subsubsection{SCANNING ELECTRON MICROSCOPY} (SEM)

A Denton Vacuum Evaporator (DV-515) was used to cover the FPC-461 and the coated and uncoated PETN samples with carbon and/or gold on a stub mounting surface. These layers were approximately 100-150 of carbon and $200 \AA$ of gold, which reduced charging the sample in the SEM.

The samples were carbon coated first in order to do SEM backscatter, but, because of insufficient charge reduction, the samples had to be gold coated. The gold coating reduced the charge sufficiently

Table 1 - SUMMARY OF EXPECTED RESULTS AND SAMPIING DEPTH OF SURFACE ANAIYTICAL TECHNIQUES

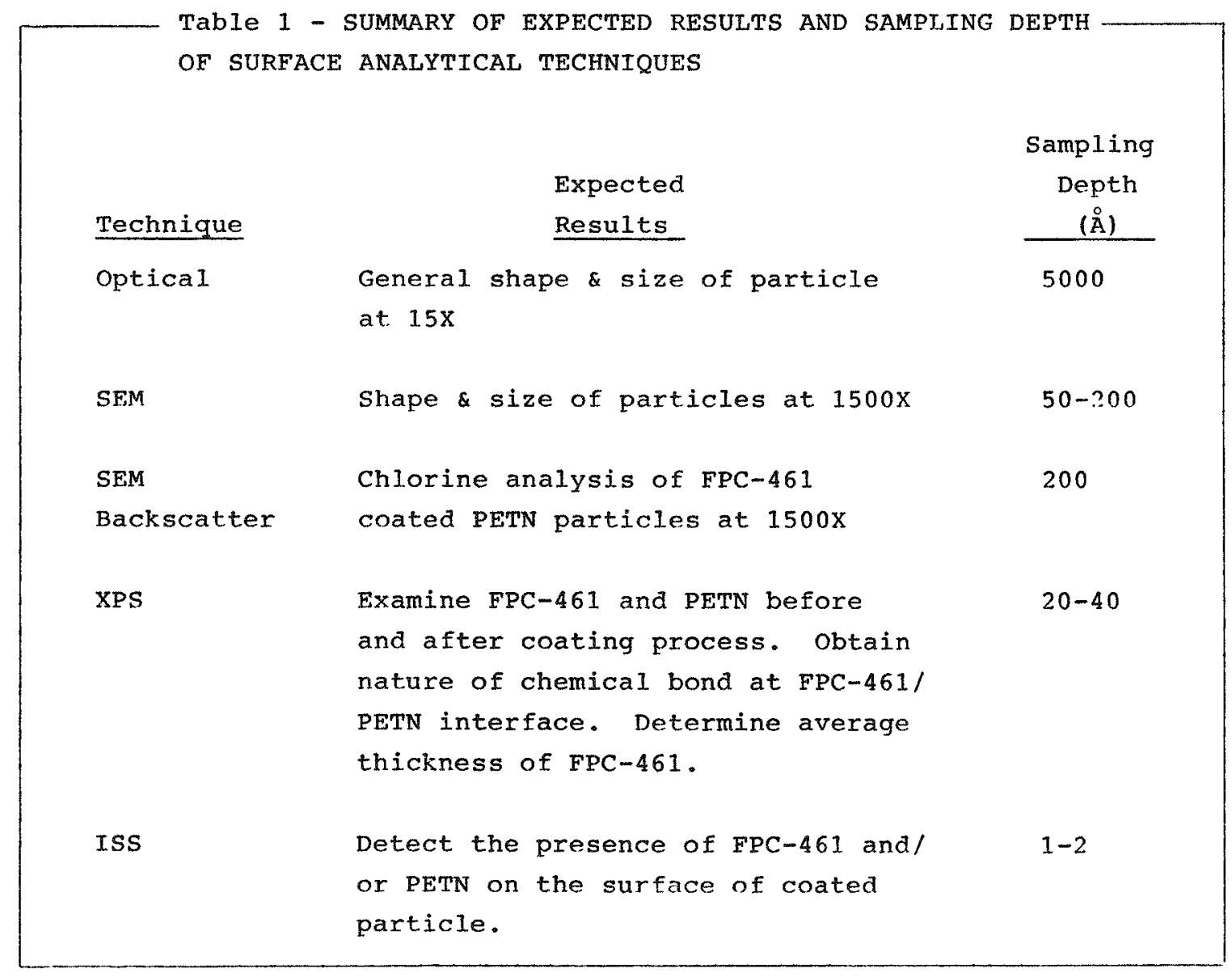


to obtain an SEM image. It then became difficult with the gold-coated samples to differentiate the chlorine of the polymer from carbon, oxygen, and nitrogen of the explosive, because of the high atomic number of gold.

A Cambridge 250 Mk2 stereoscan scanning electron microscope was used for experimentation. Chamber pressures of less than $10^{-6}$ torr were obtained, and an $11-k v$ beam was used.

\subsubsection{X-RAY PHOTOELECTRON SPECTROSCOPY} (XPS)

A Surface Science Laboratory $x$-ray photoelectron spectrometer (Model SSX-100) was used. This spectrometer is a small spot size xps analyzer capable of generating analysis regions of $150,300,600$, and $1000 \mu \mathrm{m}$. The excitation source was monochromaticized $A 1\left[K_{\alpha}\right]$ radiation. In the accumulation of these data, a spot size of $300 \mu \mathrm{m}$ was used. At this spot size, $50 \mathrm{~W}$ (that is $10 \mathrm{kV}$ or $5 \mathrm{~mA}$ ) of energy was used. A hemispherical analyzer was used in this system.

Samples were placed in front of the photon beam, and overall spectra were taken as well as individual scans. A typical time to take all the data was $30 \mathrm{~min}$ per sample. A charge neutralizer of 10-15 $\mathrm{V}$ was required to neutralize each of the samples. Duplicate runs were done on selected regions before and after analysis. No change in the spectra was noted, showing that the polymer coating and the PETN particles were not dissociated by the $A 1\left[K_{\alpha}\right]$ photons.

\subsubsection{ION SCATTERING SPECTROSCOPY (ISS)}

A Kratos ISS spectrometer system connected to a data system was used for surface characterization of the FPC-461 and the coated and uncoated PETN samples. The analyzer is a cylindrical mirror type which has a scattering angle of $138^{\circ}$. The system was pumped to pressures of $10^{-9}$ torr with an ion pump $(110 \mathrm{I} / \mathrm{s})$ and a turbomolecular pump $(330 \mathrm{I} / \mathrm{s})$. An ion beam of approximately $1 \mathrm{~mm}$ in diameter was used.

The pellet samples were mounted on a carousel and placed into the ISS sample chamber. Helium-3 was used as the scattering gas when ISS spectra were being recorded. Also, when the samples were being scanned, specific values were set the same for all scans:

1) Voltage potential $\left(E_{0}\right)=1.8 \mathrm{keV}$,

2) Multiplier $=-2.7 \mathrm{kev}$,

3) Charge neutralization $=2.1 \mathrm{~A}$,

4) Channel step size $=0.005$ energy ratio units $\left(E_{1} / E_{0}\right)$,

5) Ion current $=100 \mathrm{nA}$, and

6) Helium pressures $\cong 1 \times 10^{-7}$ torr.

Spectra were recorded within $10 \mathrm{~s}$. Within this time there was no detectable decay observed in either FPC-461 or in coated and uncoated PETN samples.

\subsection{Particle Size - Coulter Counter}

The principles of operating this instrument are given in Figure 1. The powder particle sample were dispersed in an electrolyte solution. As the electrolyte passed through the orifice, the electrodes immersed on either side detect a change in resistance caused by the particles. This generates a voltage pulse which is proportional to the size of the particle. The pulses were amplified, sized, and counted to obtain a size distribution of the dispersed particles. [2] 


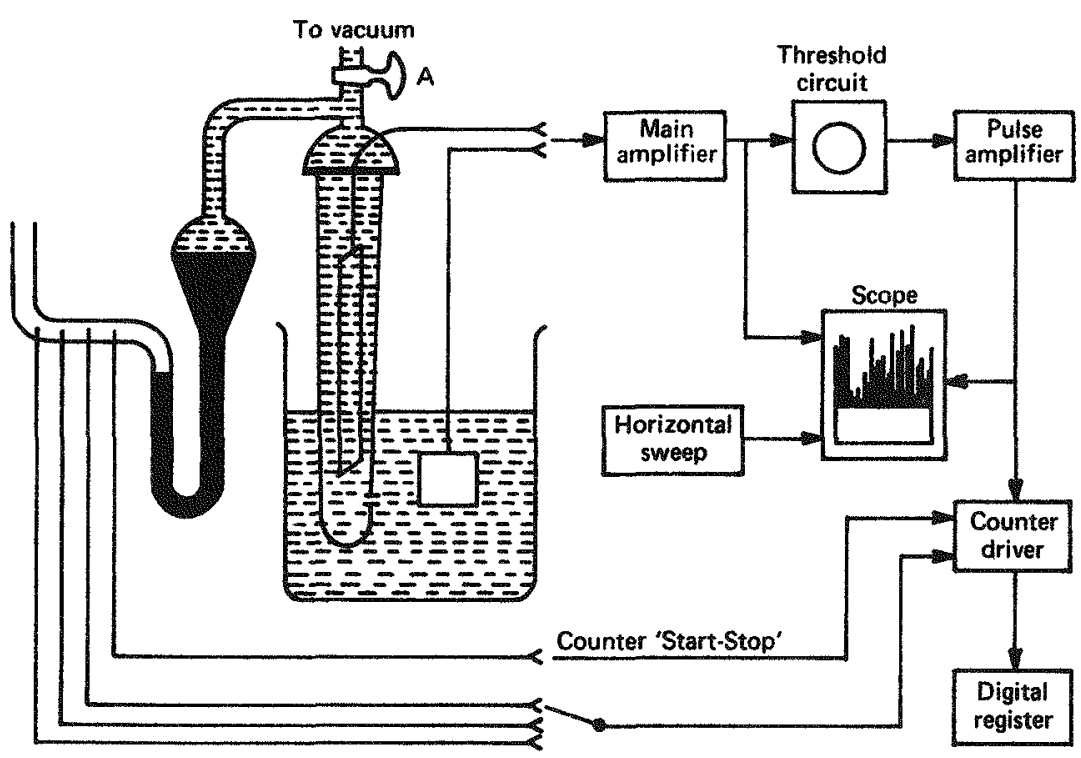

FIGURE 1 - Schematic of Coulter Counter operations.[2]

\subsection{Contact Angle Measurement}

The polymer material and the 0.5 and 20 wt 8 coated PETN powders were pressed into pellets on an Instron Model Number 1127 mechanical screw press. The pellets were pressed in a mold to $95 \%$ density to obtain aimensions of 0.748 in. in diameter and $0.083 \mathrm{in}$. thick. The coated and uncoated PETN material and the polymer material were pressed into pellets at 48,000 and $50,0001 \mathrm{~b}$ of pressure, respectively. A dwell time of $60 \mathrm{~s}$ at pressure and a ram speed of $0.2 \mathrm{in.} / \mathrm{min}$ were used.

The pellet samples wexe placed on the sample stage of a goniometer telemicroscope. Various solvents were dropped onto the pellet samples with a $1-\mathrm{mL}$ pipette. The contact angle measurements were taken from the contact angle indicator dial after the view finder was focused on the solvent drop and pellet. The various solvents ranged in surface tensions from 23.9 to 72.6 dynes/cm.

\section{Results and Discussion}

In the broadest sense, microencapsulation involves surrounding a core particle with a coating. As discussed in Appendix $A$, the coating can be used to protect the core particle from its environment, to improve handiing properties, and to contain the core particle for "timerelease" products. However, in this work, microencapsulation techniques were used to place a plastic (FPC-461) coating on an explosive (PETN) powder. The microencapsulation enhanced by starved addition process used was solvent evaporation. The microencapsulated explosive can then be prilled for further improvement of the pourability properties of the powder particles. Pourability is defined as the ease at which powder particles will flow through a Hall flowmeter (a funnel-shaped instrument).

prilling is agglomerating many particles together and involves increasing the 
stickiness of the individual microencapsulated particles so that they adhere to one another. In priliing, the weight of an average particle is therefore increased, which correspondingly increases pourability. Properties of the powder such as strength, compactibility, and integrity are increased through the prilling process. In this work, prilling was not examined, but the surface chemistry of coated PETN with a copolymer (FPC-461) was studied in detail.

In order to discuss the results of starved addition coating on the surface chemistry, two models were proposed. These two models include one in which the core particles are completely coated by the polymer (Model I), and another in which the core particles are only partially coated, but with some coating distributed on all the particles (Model II). These models are shown in Figure 2 $(A \& B)$.

The structure of PETN (pentaerythritoltetranitrate) is given in Figure 3. since PETN is an organic explosive, it is classed as a secondary explosive. Secondary explosives are less sensitive to stimulus, such as shock or heat, and are more controllable than primary explosives (inorganics such as fumates and azides). All explosive organic compounds contain nitro $\left(-\mathrm{NO}_{2}\right)$ or nitrate $\left(-\mathrm{ONO}_{2}\right)$ groups.

FPC-461 is a copolymer of vinylchloride/ trifluorochloroethylene in a $1.5 / 1.0$ weight ratio. The structure of FPC-461 is given in Figure 4.

The degree of sensitivity of the explosive (PETN) can be regulated by coating the particles with a polymer. FPC-461 was chosen for this work because it had been used on plastic bonded explosives (PBX) as well as other explosives in the industry. $[3,4]$ The intent here was to minimize the change in sensitivity of the explosive powder.

This study involved establishing whether Model I or Model II resulted from the starved addition coating of FPC-461 on PETN. These models aid in understanding changes, if any, in the surface chemistry of the polymer and explosive before and after coating, in establishing the interfacial bonding, and in discussing the wettability of coating solvents used in the microencapsulation technique.

There are certain characteristics of the surface analytical technigues that are needed to establish whether Model I or Model II is the correct hypothesis. A good surface analytical technique should (1) have good depth resolution, (2) have good spatial resolution, (3) have reasonable analyzing times, (4) be nondestructive, and (5) be able to determine the difference between FPC-46I and PETN.

Assuming that the PETN core particles are spherical, one can, to a first approximation, estimate the coating thickness for 0.5 and 20 wt $\&$ coated PETN particles. Using Equation 3.1, one can estimate the coating thickness (d) of the polymer (P) on the explosive (E), knowing the surface area of the explosive (SAE) and the density of the polymer (DP).

$$
\alpha=\frac{\frac{\text { Wt of } P}{D P}}{\text { wt of E } X S A E}
$$

where $\mathrm{DP}=1.70 \mathrm{~g} / \mathrm{cm}^{3}$ and $\mathrm{SAE}=12.600$ $\mathrm{cm}^{2} / \mathrm{g}$. The weight of polymer and explosive depends on the weight ratio of 
A) Model 1 - Totally Coated PETN Particle

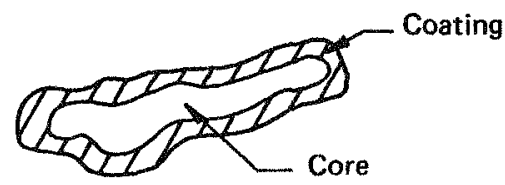

B) Model II - Partially Coated PETN Particle

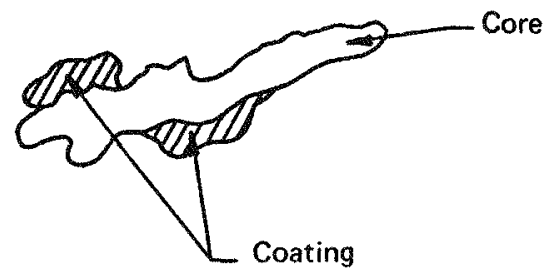

FIGURE 2 - Manner in which particles might be coated.

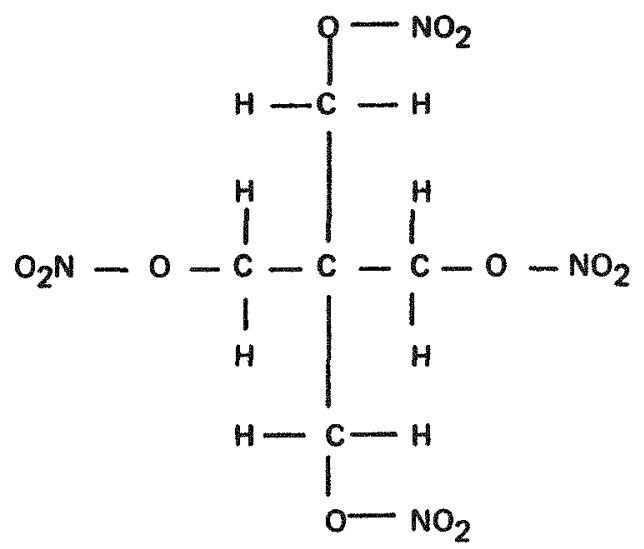

FIGURE 3 - Chemical structure of PETN.

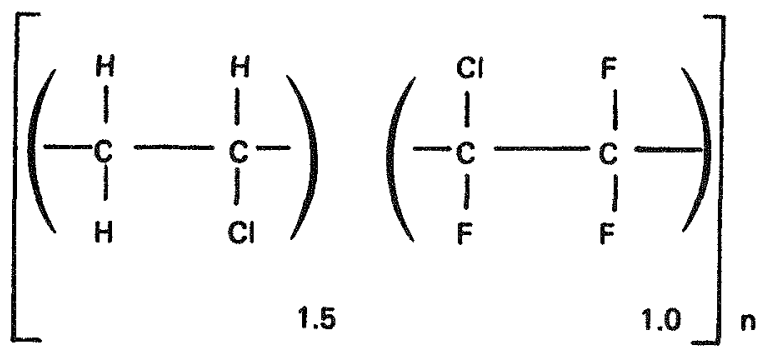

FIGURE 4 - Chemical structure of FPC-461. polymer to explosive used in coating; for example, the $0.5 / 99.5$ in the 0.5 wt o coating and the $20 / 80$ in the 20 wt 8 coating. Results indicate that $\mathrm{a} \cong 2.3 \AA$ and $d \cong 1200 \AA$ for 0.5 and 20 wt $\%$ coatings, respectively. Thus, the surface analytical techniques should be able to examine coating thicknesses on the order of $20 \AA$ in the case of 0.5 wt $\%$, and $1200 \AA$ in the case of 20 wt 8 coated PETN. The results of each of the analytical techniques discussed in the following paragraphs are in light of the proposed models.

Optical microscopy is a good overall technique for analysis of polymer and explosive particle shapes and sizes. This can be seen in the photographs of Figure 5 (A-D). The photographs also show, however, that the optical microscope does not have sufficient resolving power for surface characterization of the polymer on the explosive. Therefore, optical microscopy is not suited for this work but is included only for completeness.

The scanning electron microscope (SFM) gives much better spatial and depth resolution images than optical results. The SEM images can be seen in the photographs of Figure $6(A-D)$. It is a little difficult to see the polymer coating (FPC-461) on the core particles (PETN) of the 0.5 wt $\frac{a}{2}$ coated explosive, but the polymer coating is shown draping across the core particles of the 20 wt 8 coated material. The photographs of the 20 wt 8 coated PETN show that the polymer coating is distributed over the core particles.

The detection of chlorine in the SEM backscatter mode would indicate the presence of polymex on the explosive 


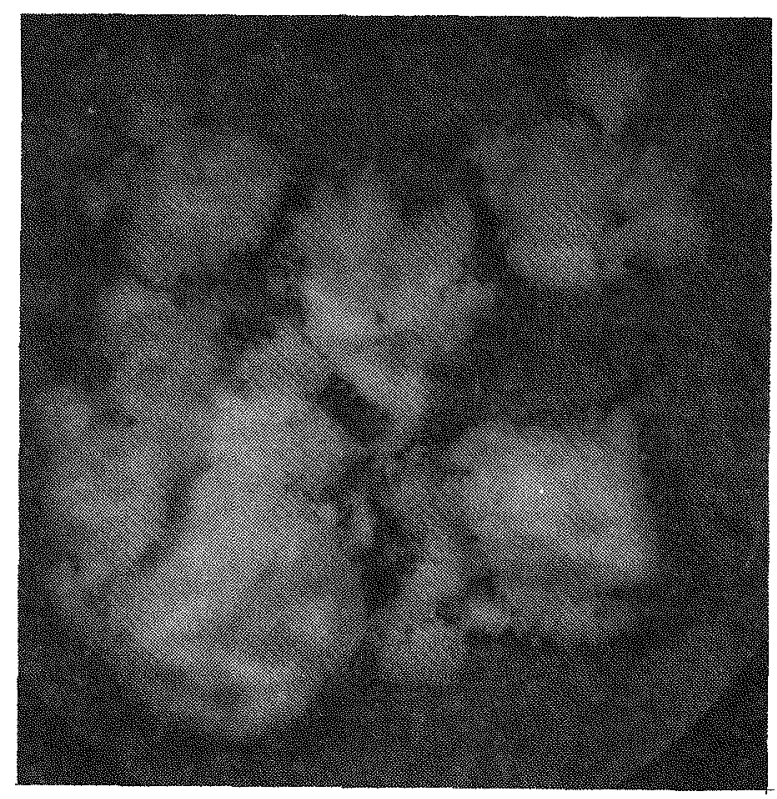

(a) polymer

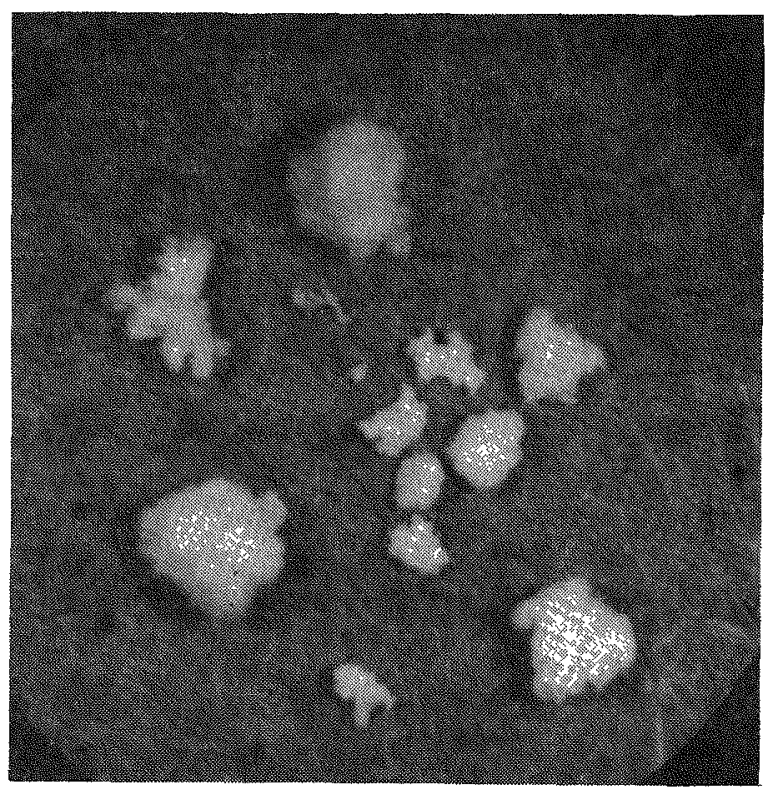

(c) $0.5 \%$ coated PETN particles

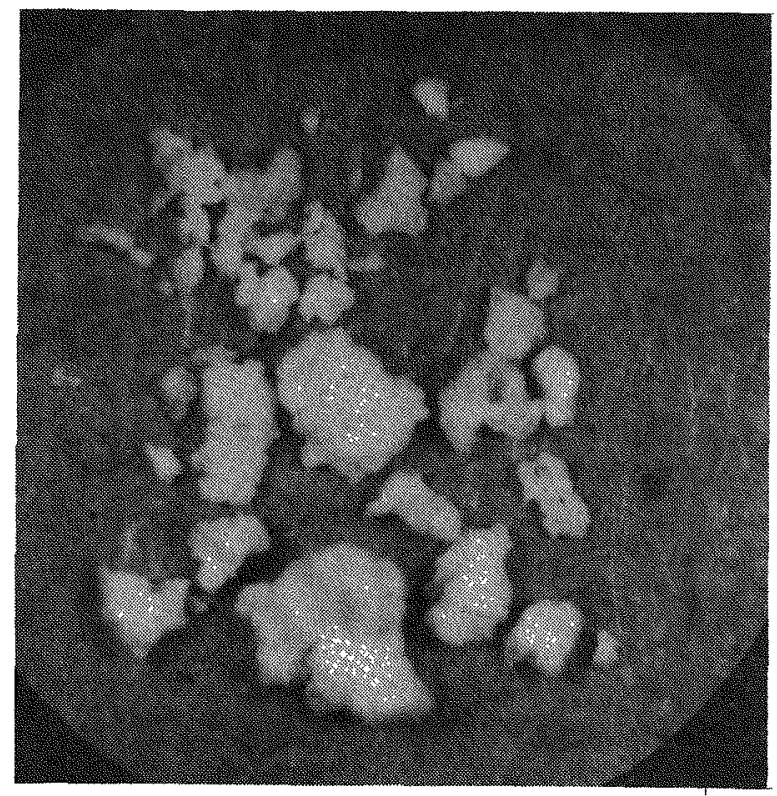

(b) PETN particles - no coating

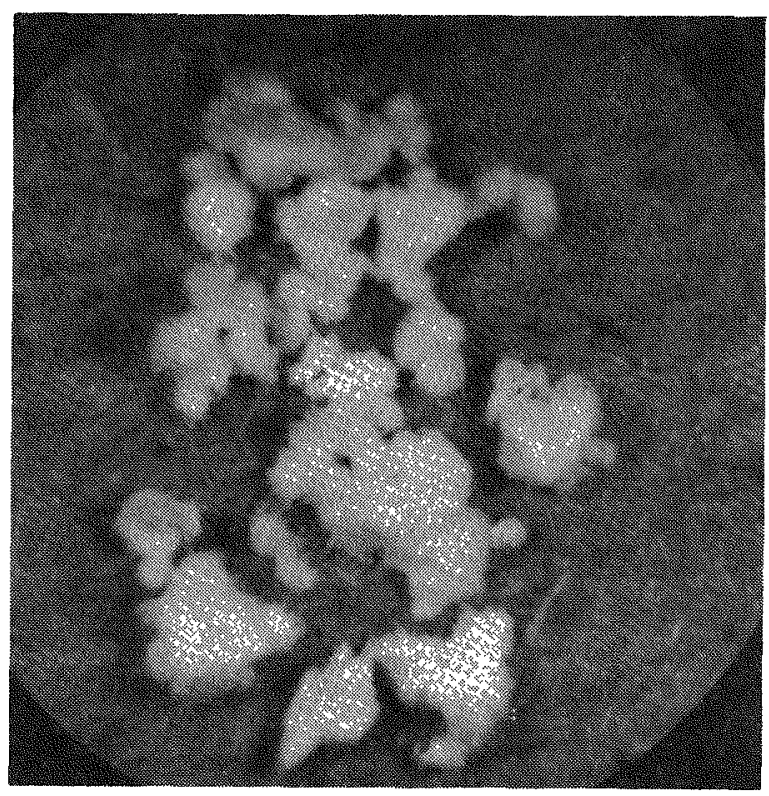

(d) $20 \%$ coated PETN particles

FIGURE 5 - Optical microscopy photographs (at 15X). 


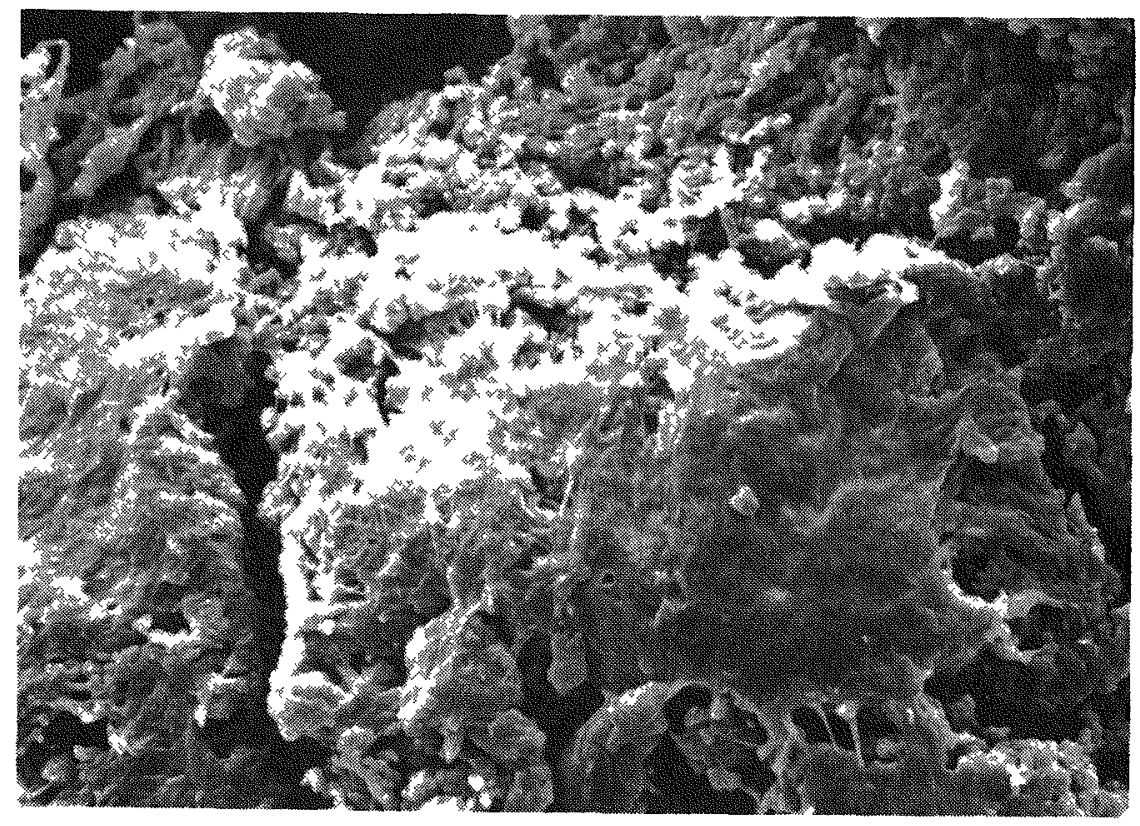

(A) - Polymer.

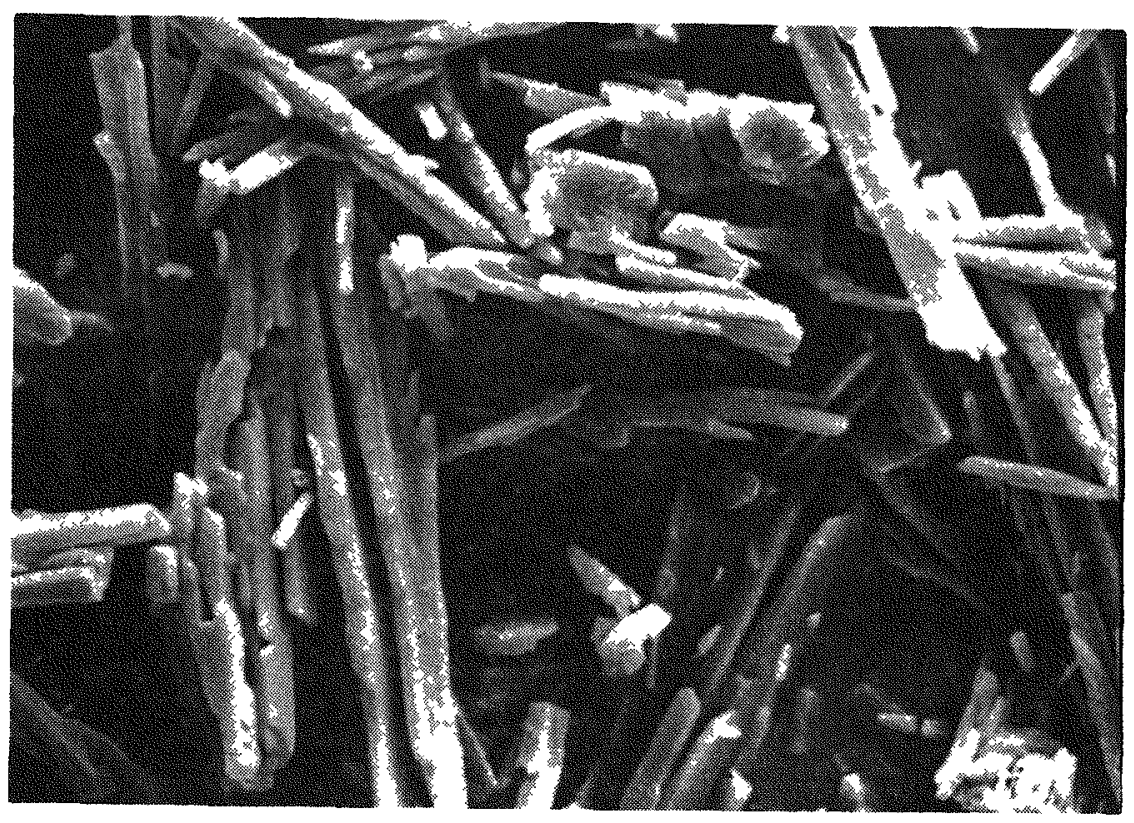

(B) - PETN particies-no coating.

FIGURE 6 - Scanning electron microscopy photographs (at 1500x). 


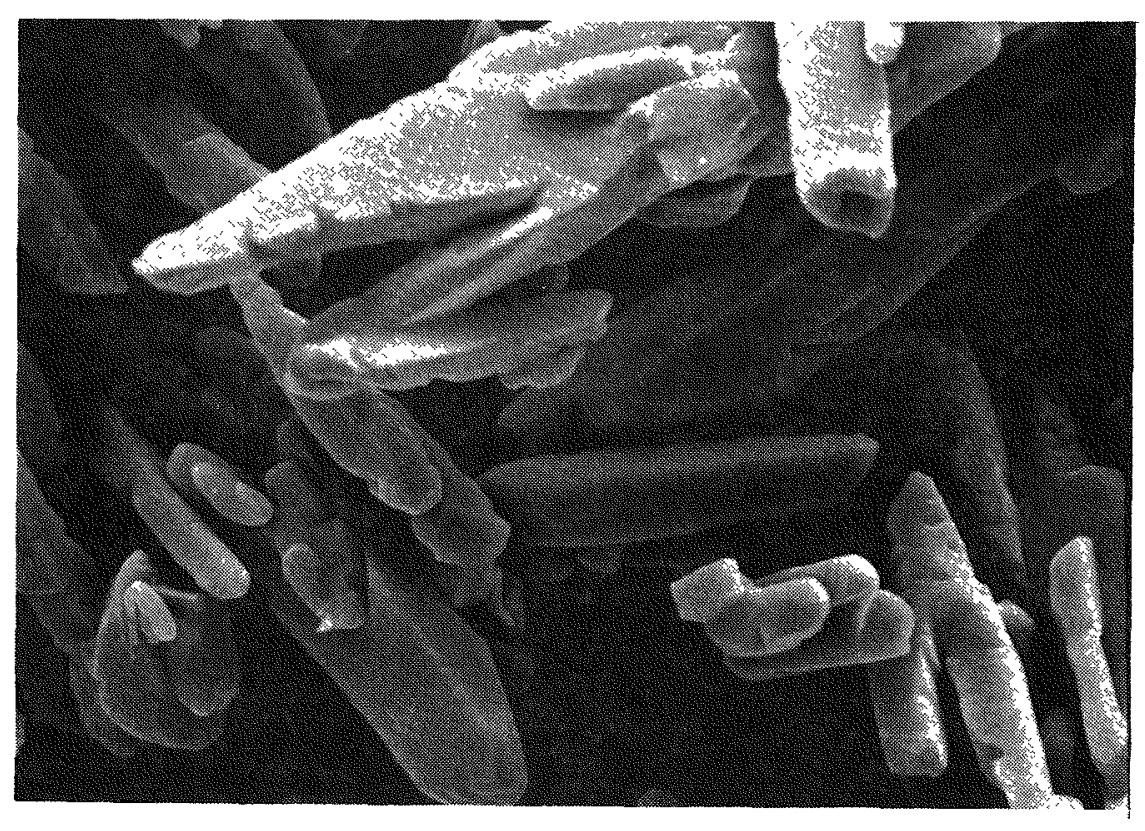

(C) $-0.5 \%$ coated PETN particles.

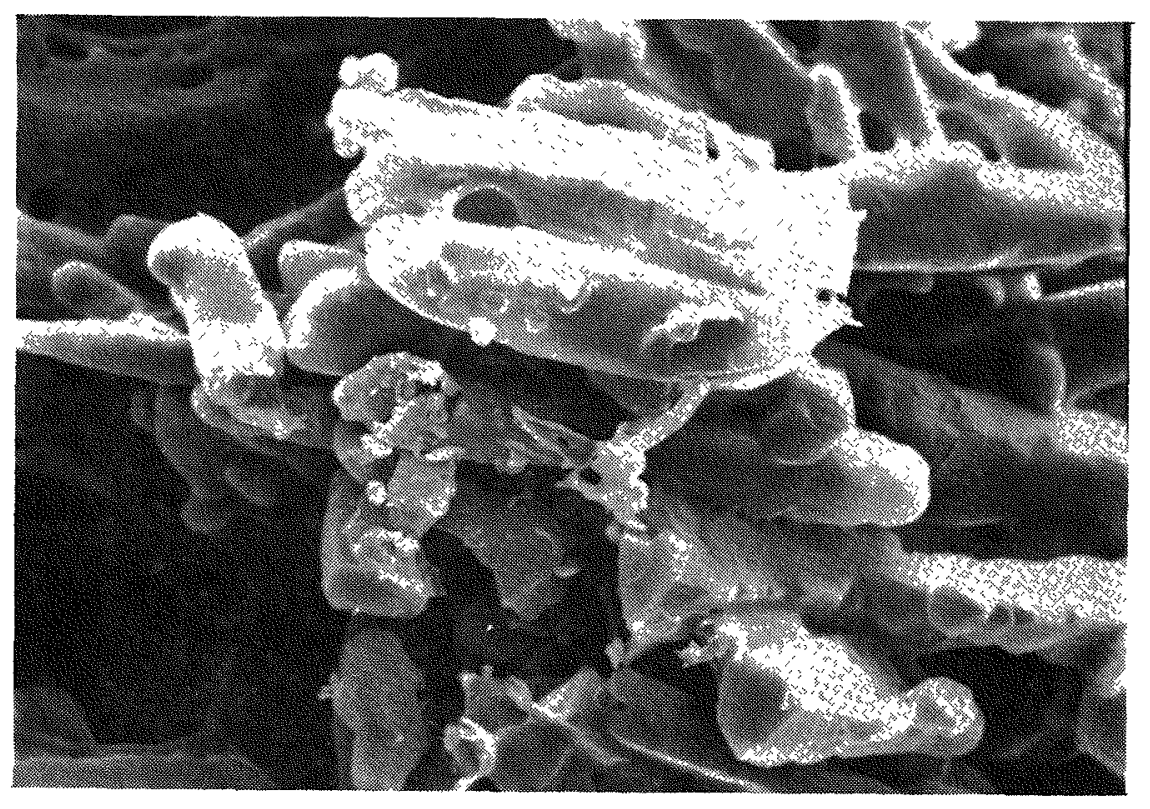

(D) - $20 \%$ Coated PETN particles.

FIGURE 6 - Continued. 
surface. If chlorine had been evenly distributed on the sample surface, then Model I would have been chosen to support the microencapsulation enhanced with starved addition hypothesis. SEM backscatter also has excellent spatial resolution on the order of $5,000 \AA$. Because of the charging (of the sample) problems, this technique could not successfully be completed.

X-ray photoelectron spectroscopy (XPS) has excellent depth resolution but poor spatial resolution. Poor spatial resolution means that many particles are analyzed at any one time. But, XPS does have the capabilities to distinguish between the coating (FPC-461) and the explosive (PETN) particles. The overall spectra of FPC-461 and the coated and uncoated PETN samples are shown in Figure 7 (A-D). These data show the elements of carbon, nitrogen, and oxygen characteristic of PETN and carbon, fluorine, and chlorine characteristic of FPC-461. (XPS has difficulty in characterizing the hydrogen electron because the binding energy of the electron is very close to that of the valence electrons.) Thus, characteristic XPS Iines from both FPC-461 and PETN are seen. The average depth of analysis is 20-40 . If it is assumed that there is a uniform coating on the core particles, the average coating thickness can be estimated by the following equation.

$$
I_{\text {PETN }}=I_{\text {PETN }} e^{-d / \lambda}
$$

where $I_{\text {PETN }}$ is the intensity of an XPS photoelectron 1 ine from the explosive which has been coated.

$I_{\text {PETN }}$ is the intensity of an XPS photoelectron line from the uncoated explosive $(d=0)$. $\lambda$ is the mean free path of an XPS photoelectron line, and

d is the coating thickness.

If it is assumed that $\lambda=20-40 \AA$, and that the coating is uniformly surrounding the explosive, the calculation of the coating thickness reveals $\mathrm{d}=3-6 \stackrel{\circ}{\mathrm{A}}$. This calculation of coating thickness is different from that of $23 \AA$ obtained using the Fisher subsieve surface area value of $12,600 \mathrm{~cm}^{2} / \mathrm{g}$. This difference could be due to the irregularity of the particle surface. The more irregular the surface of the particle, the higher the actual surface area of the particle. These data indicate that possibly the surface area of the explosive is larger than $12,600 \mathrm{~cm}^{2} / \mathrm{g}$. If this were true, then there would be a much thinner, more evenly distributed coating on the particle.

Figure 8 (A-D) shows overall ISs spectra of the coating and of uncoated and coated PETN pellets. These data were recorded within $600 \mathrm{~s}$ after the pellets were exposed to the ion beam. Also included within each of the figures are inserts of the nitrogen, oxygen, and fluorine regions, as measured on a fresh part of the sample; these data were recorded within $24 \mathrm{~s}$. One will immediately note that the fluorine-to-oxygen signal. intensities are higher in the insert spectra for both the 0.5 and 20 wt 8 samples, as compared to the overall scans. This is due to ISS beam damage effects which decompose or sputter the polymer coating from the explosive. More decomposition occurs at longer irradiation times.

The time required to decompose 108 ( $\left.t_{10}\right)$ of the explosive was measured as $124 \mathrm{~s}$. 


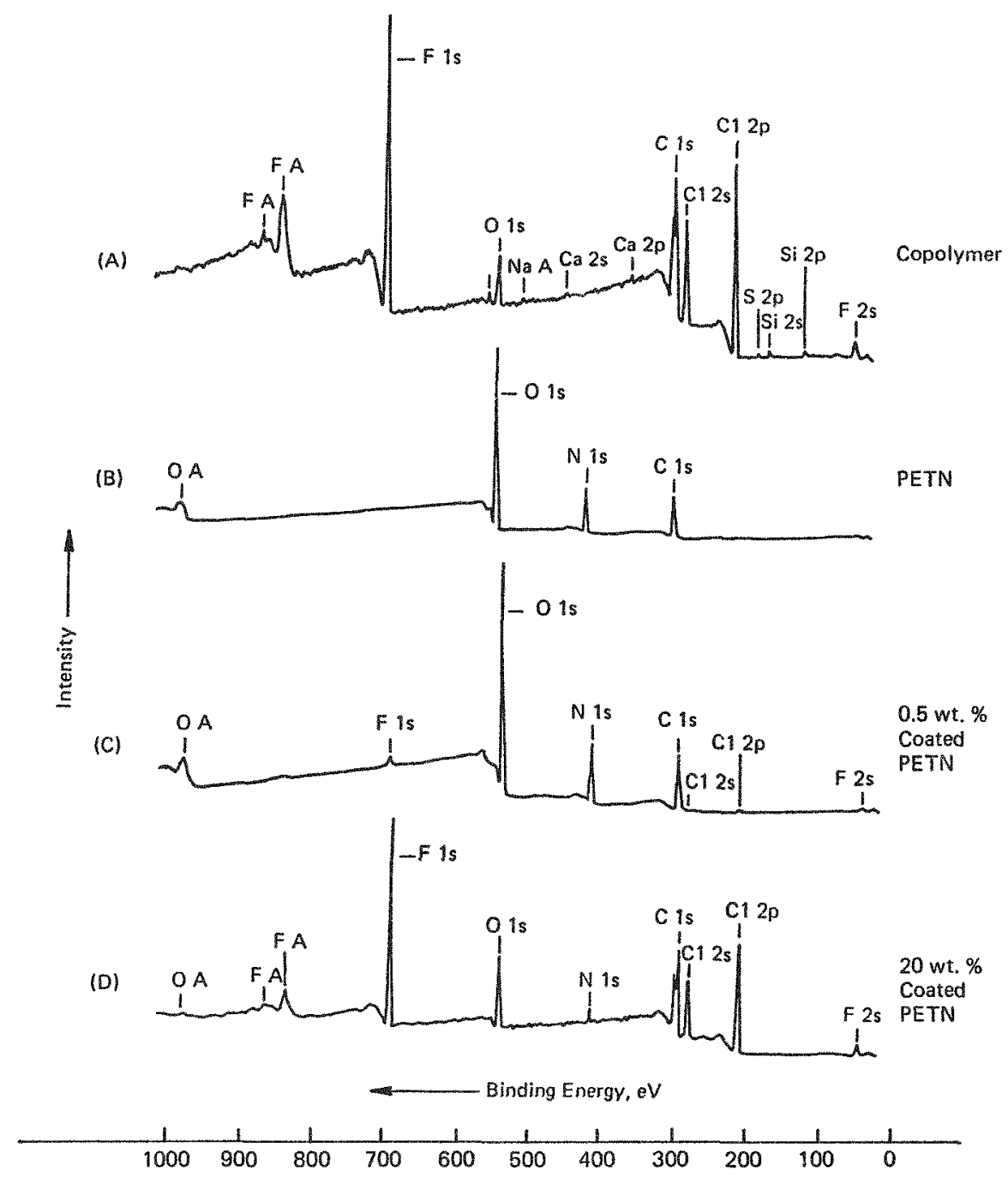

FIGURE 7 - X-Ray photoelectron spectroscopy overall spectra of (A) polymer,

(B) PETN-no coating, (C) 0.5 wt \% coated PETN, and (D) 20 wt \% coated PETN.

The ISS fluorine and chlorine signals of the polymer did not decrease during $375 \mathrm{~s}$ of ion beam exposure. However, polymer decomposition was observed on both 0.5 and 20 wt $s$ coated PETN specimens; $t_{10}$ was measured to be $40 \mathrm{~s}$ for these specimens. Thus, the insert spectra of Figure 8 (A-D) represent scans taken with little or no detectable ISS beam damage of the explosive or polymer during data analysis. Therefore, it can be concluded that the explosive is indeed present on the particle surface of the 0.5 wt 8 coated PETN specimen; but probably not on the 20 wt 8 coated PETN specimen. These results are in support of Model II (a partially coated particle). If Model I (totally coated particle) had been the case, only the coating elements (C, $F$, C1) and not the explosive particle elements (C, N, O) would appear in the spectra.

Equation 3.2 can now be rewritten to include partially coated specimens; that is: 


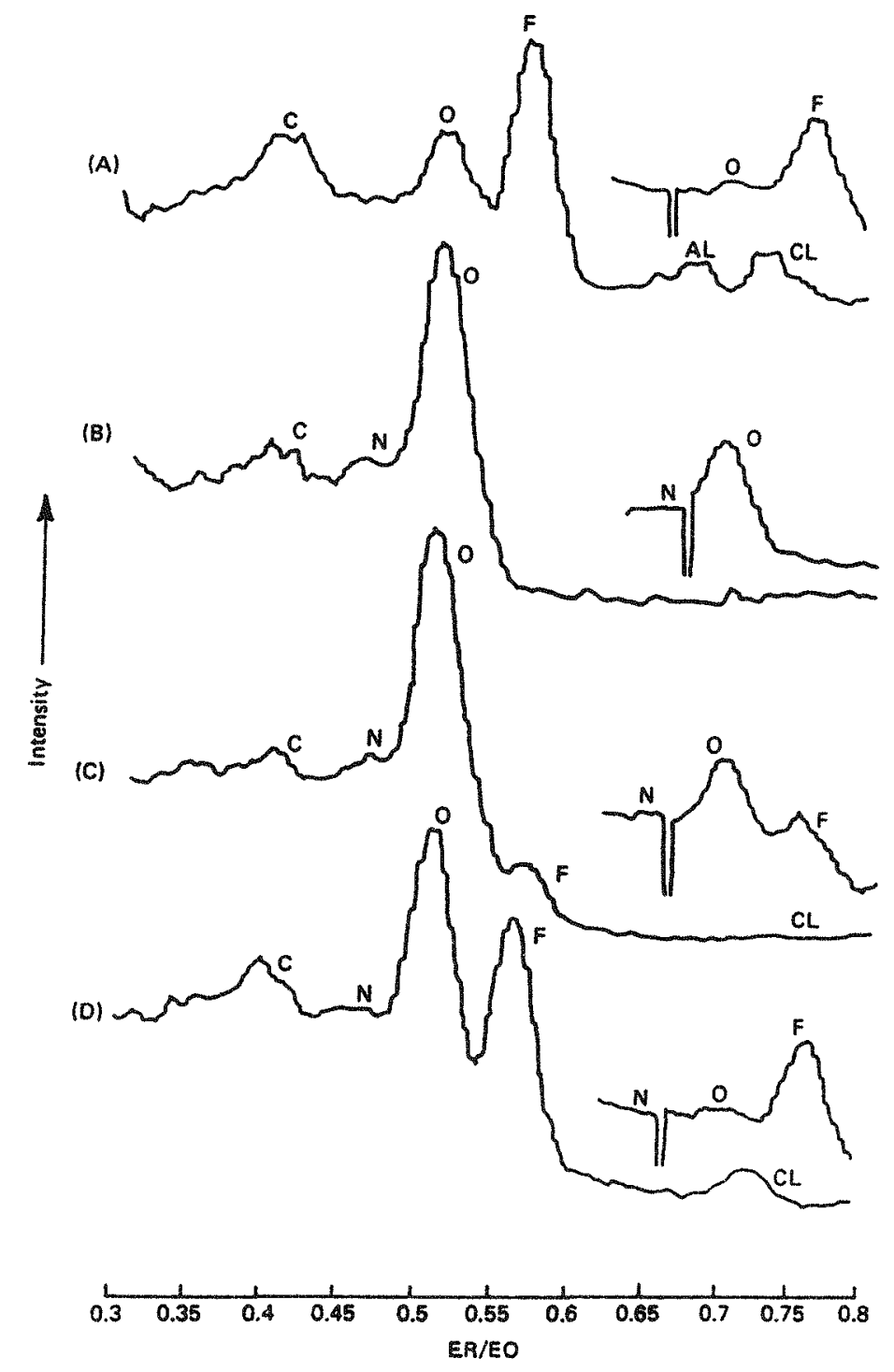

FIGURE 8 - Ion scattering spectroscopy spectra of (A) polymer pellet, (B) PETN pellet-no coating, (C) 0.5 wt \% coated PETN pellet, and (D) 20 wt \% coated PETN pellet.

$I_{\text {PETN }}=\frac{P}{100} I^{*}{ }_{\text {PETN }} e^{-d / \lambda}+$

$\frac{\mathrm{E}}{100} \mathrm{I}^{*}$ PETN

where $P$ and $E$ are the percentage of polymer and explosive, respectively, observed on the surface of coated specimens. $P$ and $E$ are obtained from the ISS data. $P$ and $E$ have been measured for 0.5 wt 8 and found to be 44 and 56 , respectively. Isikewise for 20 wt $8, P<768$ and
$E>24 \%$. Calculation of the coating thickness (d) for $\lambda=20$ to $40 \AA$ showed it to be 6 to $12 \AA$ thick in the coated regions for the 0.5 wt 8 coated PETN material, in better agreement with that calculated from surface area measurements. The calculations show the 20 wt 8 cnated PETN specimen to be a minimum of $44 \AA$ for $a \lambda$ of $20 \AA$ and $88 \AA$ for $a \lambda$ of $40 \AA$. Once the thickness of the coating becomes twice the average analysis depth 
(2 $\lambda)$, estimation of $d$ becomes very poor, and only minimum values of thickness can be given.

The results of the surface characterization by the above surface analytical techniques indicate that the microencapsulation enhanced with starved addition process of this study conforms to the hypothesis of Model II. The explosive particles are only partially coated. However, this is sufficient to achieve the desired effect. The coated powders do show increased flow (pourability) of the powder particles, which improves the efficiency in production of the final. product.

\subsection{Bonding in Microencapsulation Enhanced with Starved Addition}

The bonding of electrons in atoms of polymers and explosives can be determined by measuring the XPS binding energy to $\pm 0.2 \mathrm{ev}$.

The nitrogen (1s), oxygen (1s), fluorine (1s), and chlorine (2p) binding energies were determined for the polymer, the core (PETN) explosive, and the coated PETN materials. These data are illustrated in Figures $9(A-D)$ through $12(A-D)$.

Table 2 summarizes the results of the binding energies. The binding energy of nitrogen in coated and uncoated PETN samples is $408.2 \mathrm{eV}$. This is characteristic of a nitrogen atom bound to three oxygen atoms such as in the nitrate linkage. More important is the fact that the nitrogen is the same for all specimens. This indicates that during the microencapsulation enhanced with starved addition process, nitrogen is not changing in oxidation state. Likewise, the oxygen (1s) of the explosive does not vary with the amount of coating. The chemistry of the oxygen in coated and uncoated specimens is also not chanding during the starved addition process.

The binding energies of fluorine and chlorine also show no change to the polymer during microencapsulation enhanced by starved addition. An aboveambient temperature $\left(45^{\circ} \mathrm{C}\right)$ is involved in the process. Since no change in the binding energies occurred, heating did not change the oxidation state of the elements of FPC-461 nor of PETN. Since the nitrogen, oxygen, fluorine, and chlorine are not showing binding energy changes, it can be concluded that both PETN and FPC-461 do not involve a change in oxidation state during the coating process. Therefore, the bonding between the polymer and the explosive must involve either a mechanical attachment of the coating on the particle or a weak Van der Waals force.

On examining the two proposed models and the results of the surface analytical techniques, one notes that the type of bonding involved between the coating and the PETN particle is mechanical. This is concluded because the electronegative groups of the coating and the PETN particle prevent the attractive forces that are necessary for a Van der Wals type bond to occur.

The mechanical bond occurs when the coating grabs onto the PETN particle at the irregularities in the surface. Figure 13 illustrates how the mechanical bond between the coating and the PETN particle might occur. The bond may also be achieved by the plastic shrinking over 


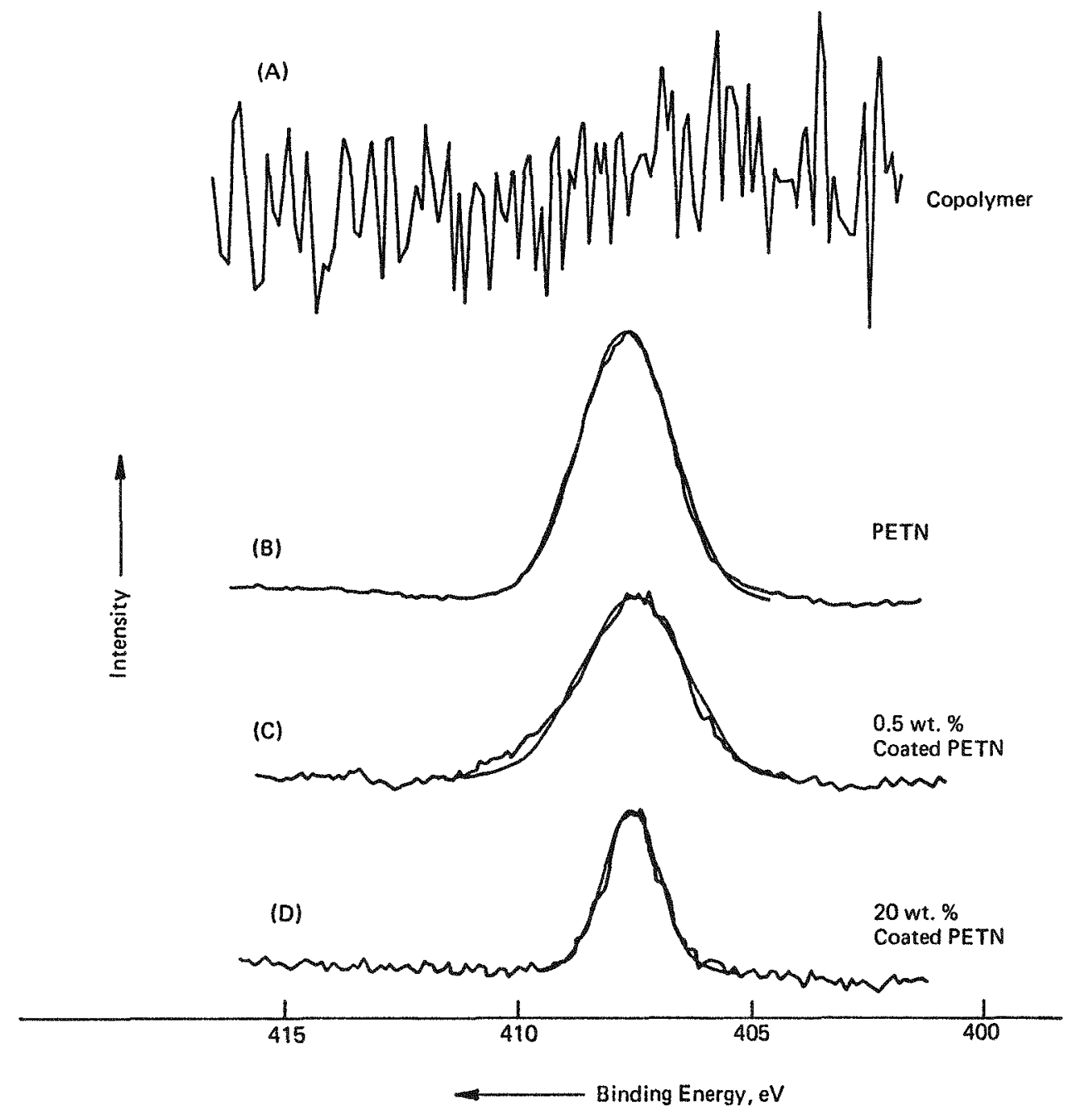

FIGURE 9 - N(1s) XPS spectra for (A) polymer, (B) PETN-no coating, (C) 0.5 wt $\%$ coated PETN, and (D) 20 wt \% coated PETN.

the surface. This figure also portrays how much the surface area of a particle can be increased as a result of surface irregularities.

\subsection{Surface Wettability}

Ideal conditions for microencapsulation enhanced with starved addition of PETN with FPC-461 include: (1) a solvent that dissolves the polymer but not PETN, (2) PETN particles that suspend in the media, but are not soluble in the media, (3) good wettability of the polymersolvent solution on the PETN particles,
(4) a uniform distribution of the polymer on the PETN (this will allow for prililing-particle agglomeration), and (5) processing temperatures that do not change the chemistry of the PETN particles or cause cracking of the coating during the evaporation of the solvent or the drying process.

The conditions of this coating study were similar to those above. FPC-461 is not soluble in many solvents, but the polymer was found to be slightly soluble in the methylene chloride, which was, therefore, 


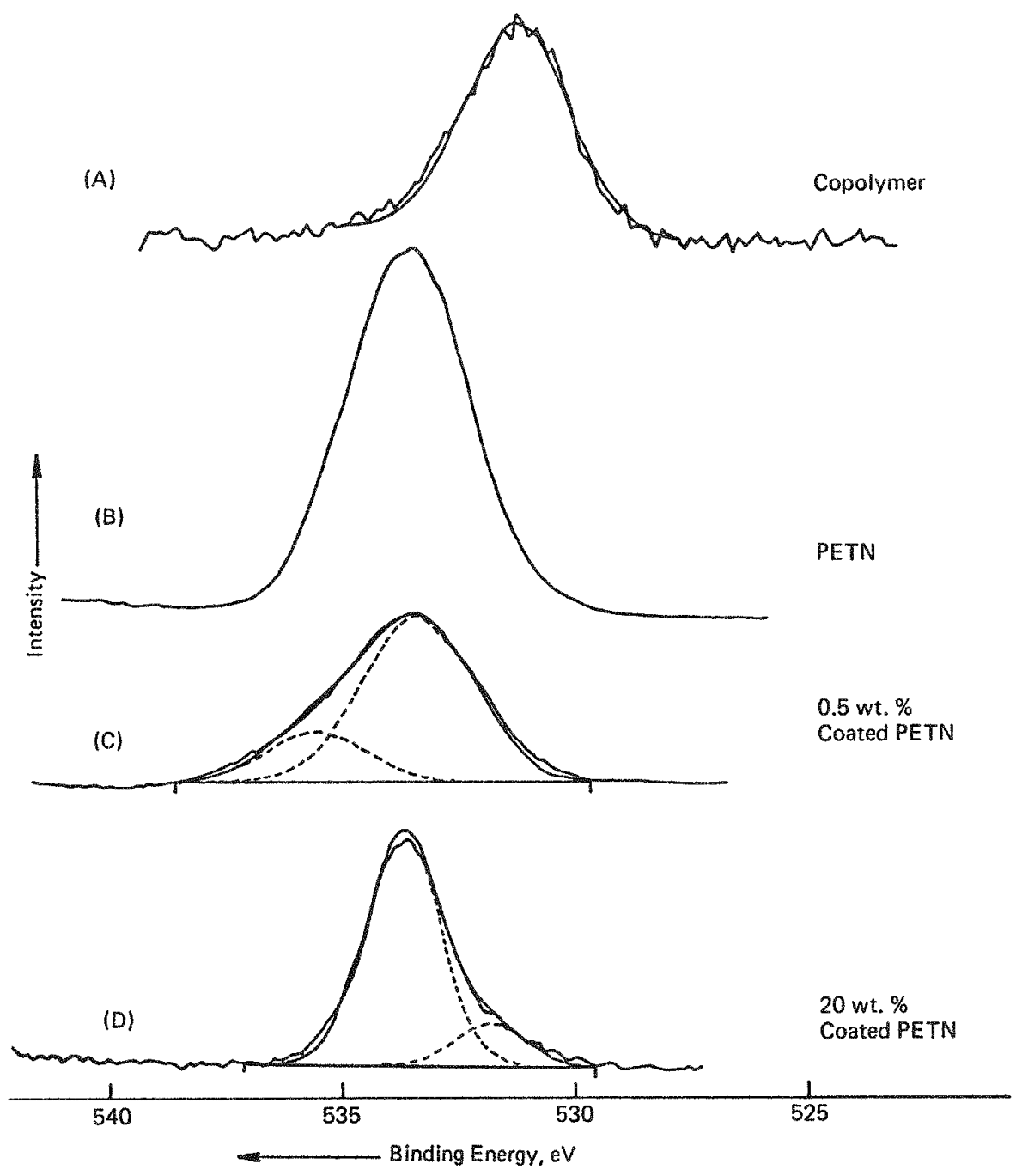

FIGURE $10-0(1 \mathrm{~s})$ XPS spectra for (A) polymer, (B) PETN-no coating, (C) 0.5 wt $\%$ coated PETN, and (D) 20 wt \% coated PETN.

chosen for use in polymer-solvent starved addition. PETN is not soluble in this solvent nor in the suspending media. The processing temperatures used were quite mild $\left(45^{\circ} \mathrm{C}\right)$, and there did not seem to be any direct evidence from optical and SEM studies of cracks being formed in the coating. (Cracks were found within a few areas of the 20 wt $\%$ coated PETN, but these were attributable to vacuum drying in the SEM instrument.) The XPS spectra show that there was no change in the chemistry of the PETN particles, nor was there a change in the FPC-461 before and after the coating process. The SEM photographs of the 20 wt $\%$ coated PETN also indicate that there is a fairly good distribution of polymer on the PETN particles.

Equation (7) of Appendix $C$ indicates that acceptable wettability requires low values for the contact angle, and thus, low values for the surface tension between the Iiquid and the vapor (atmosphere). The contact angle for each of the various solvents of Table 3 was measured on a solid surface (pressed 

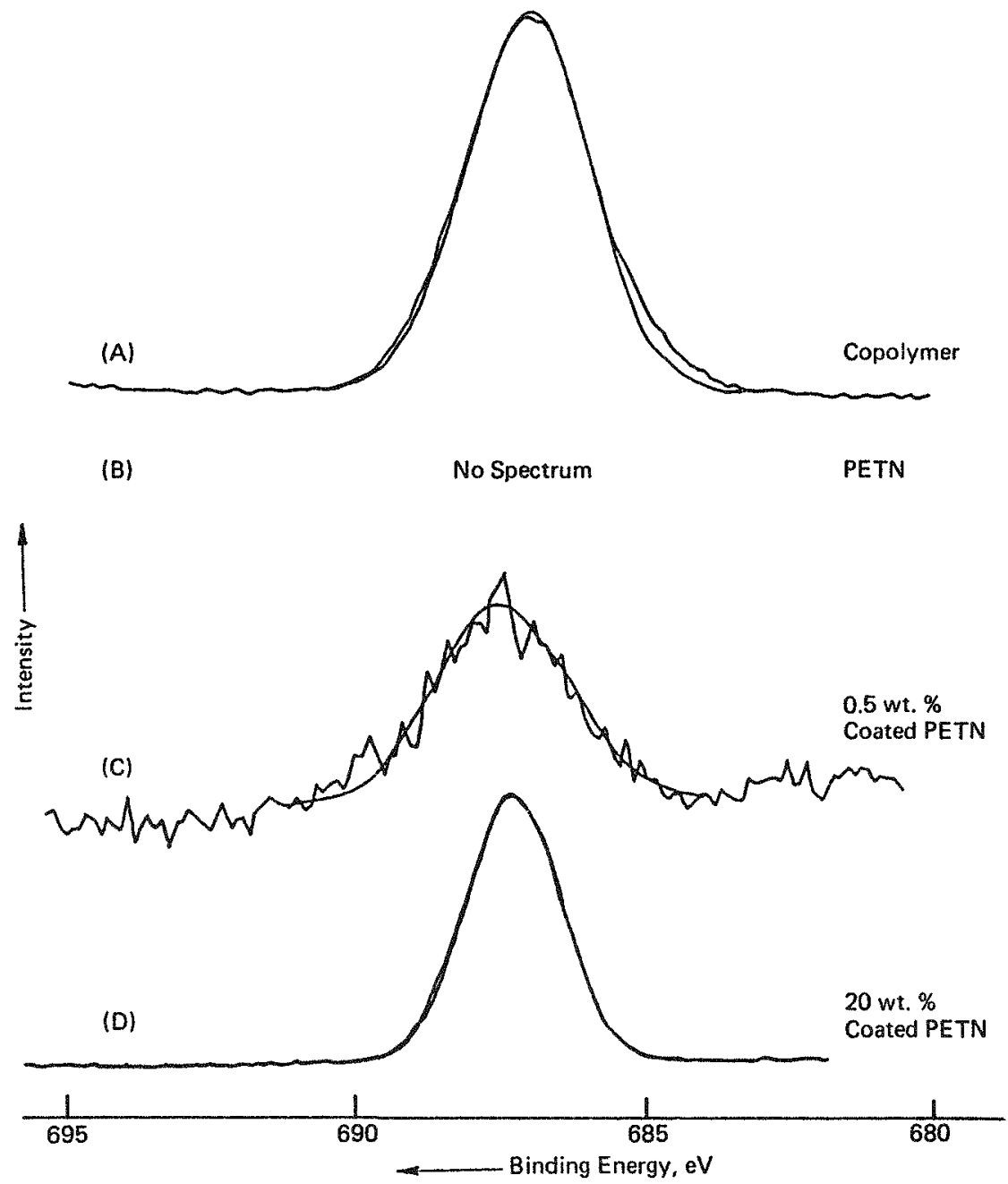

FIGURE 11 - $F(1 s)$ XPS spectra for (A) polymer, (B) PETN-no coating, (C) 0.5 wt \% coated PETN, and (D) 20 wt \% coated PETN.

pellet). Wettability of the solvents on the FPC-461 and on the coated and uncoated PETN samples, as well as the critical surface tension ( $\partial_{c}$ ) of these materials, can be determined from the contact angle measurement. The critical. surface tension determines the maximum value at which wettability can be obtained. Values that are hiqher than the critiral surface tension indicate a surface that will not wet.

One method to obtain $\partial_{C}$ is by a zisman plot. A zisman plot is a graph of the cosine of the contact angle lof the various solvents on the pelletsl as a function of the surface tension of the solvent. The wettability of each solvent on the smooth and rough surfaces of the FPC-461 and on the coated and uncoated PETN is recorded in the $\mathrm{zisman}$ plots as shown in Figure $14(A-G)$. A line which best fits the data is drawn through the plot using the least squares method. A line is then arawn parallel to the $y$-axis (ordinate) from the point where the line crosses at $\cos \theta=1$ to the point on the $x$-axis (abscissa). The point on the 
(A)
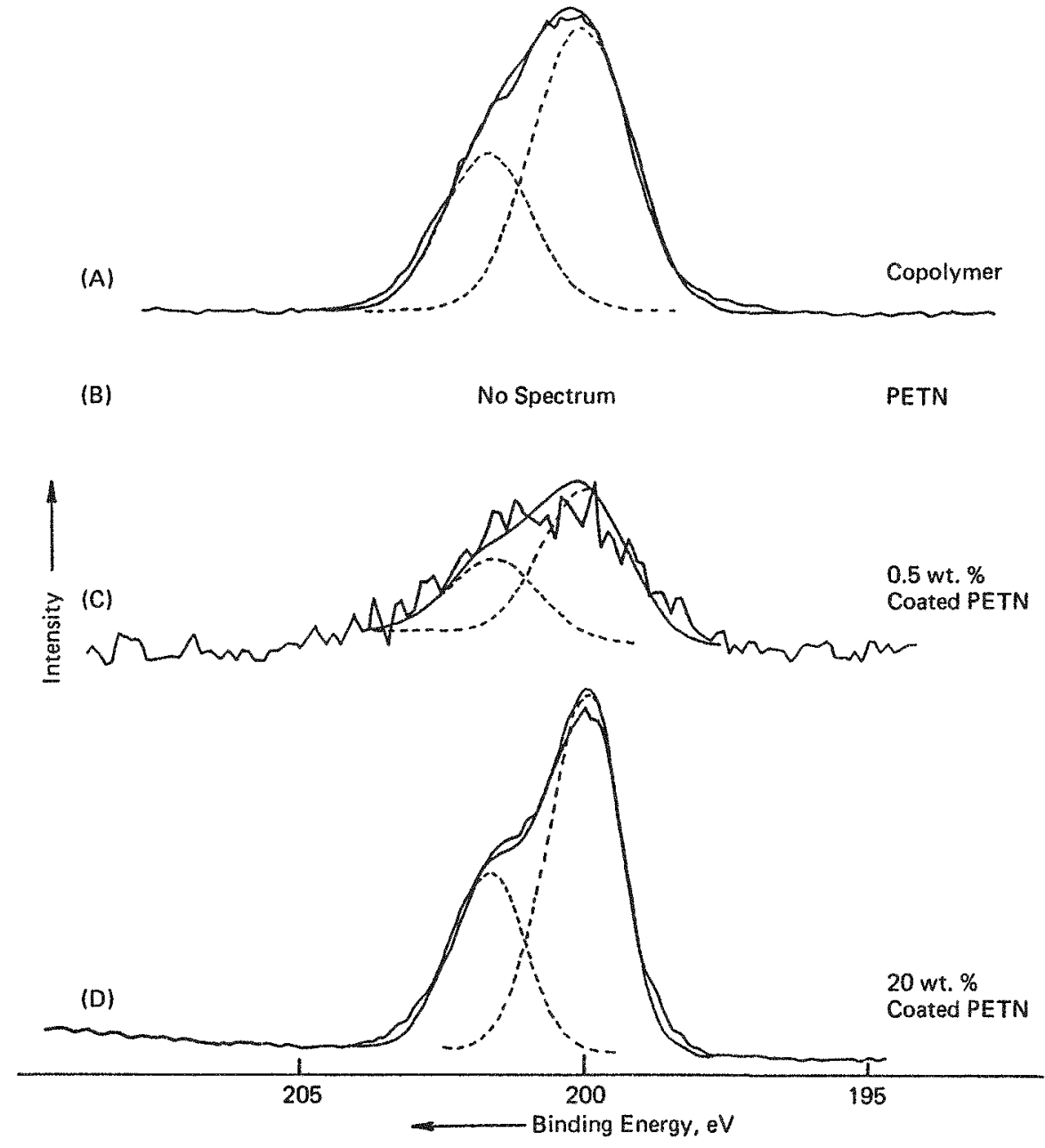

FIGURE 12 - CI(2p) XPS spectra for (A) polymer, (B) PETN-no coating, (C) 0.5 wt $\%$ coated PETN, and (D) 20 wt \% coated PETN.

Table 2 - SUMMARY OF BINDING ENERGIES FROM THE XPS SPECTRA

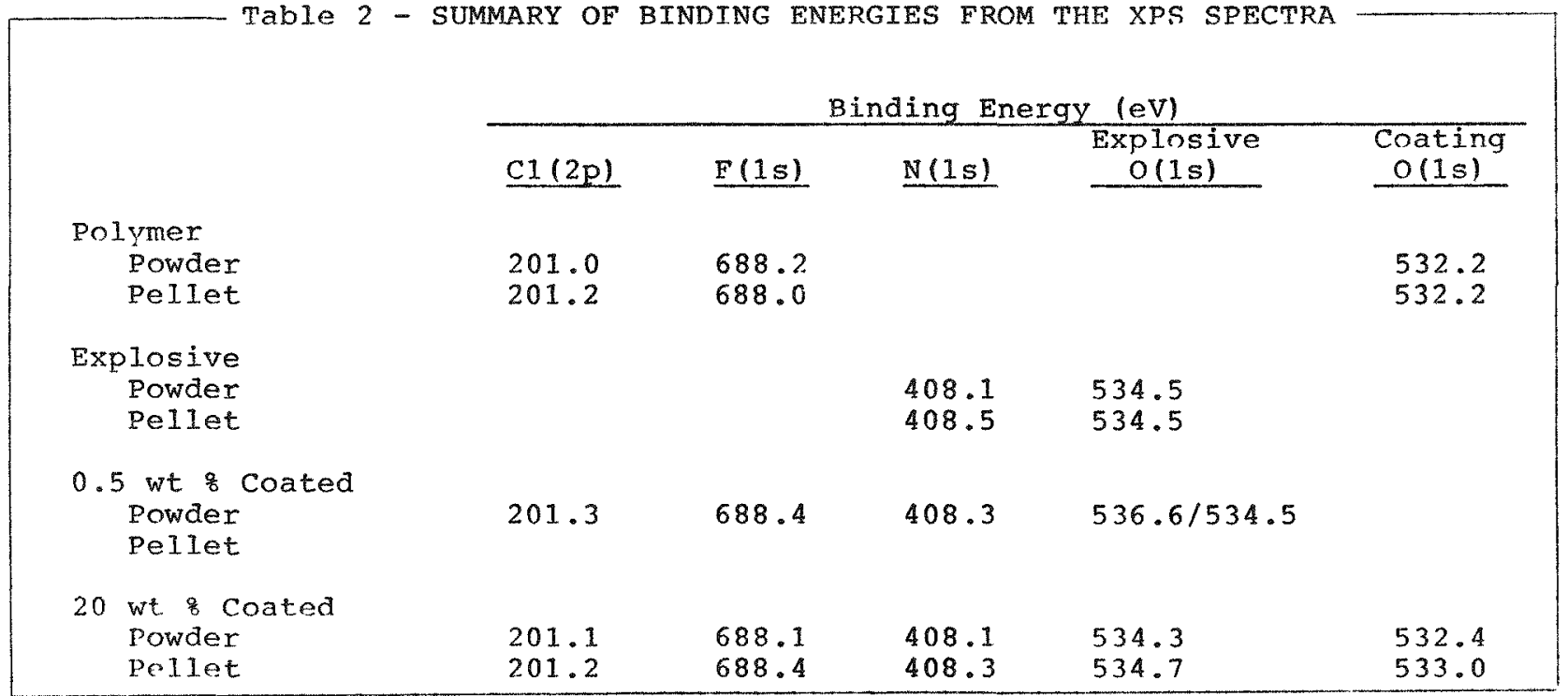




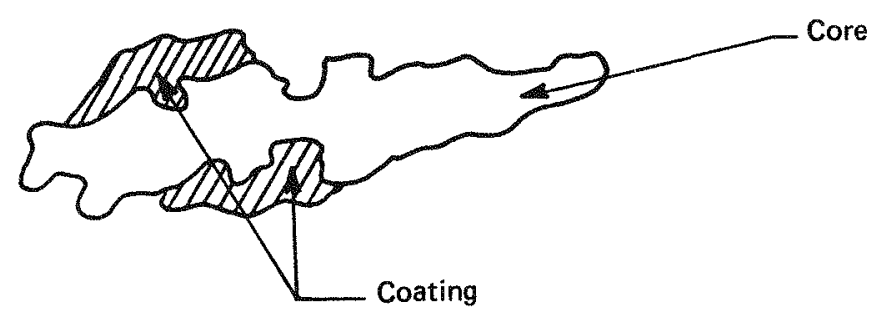

FIGURE 13 - I11ustration of mechanical bond between FPC-461 and the PETN particle.

Table 3 - CONTACT ANGLE MEASUREMENTS OF VARIOUS SOLVENTS ON THE POLYMER AND THE UNCOATED SAMPLES OF PETN (SMOOTH PELLETS)

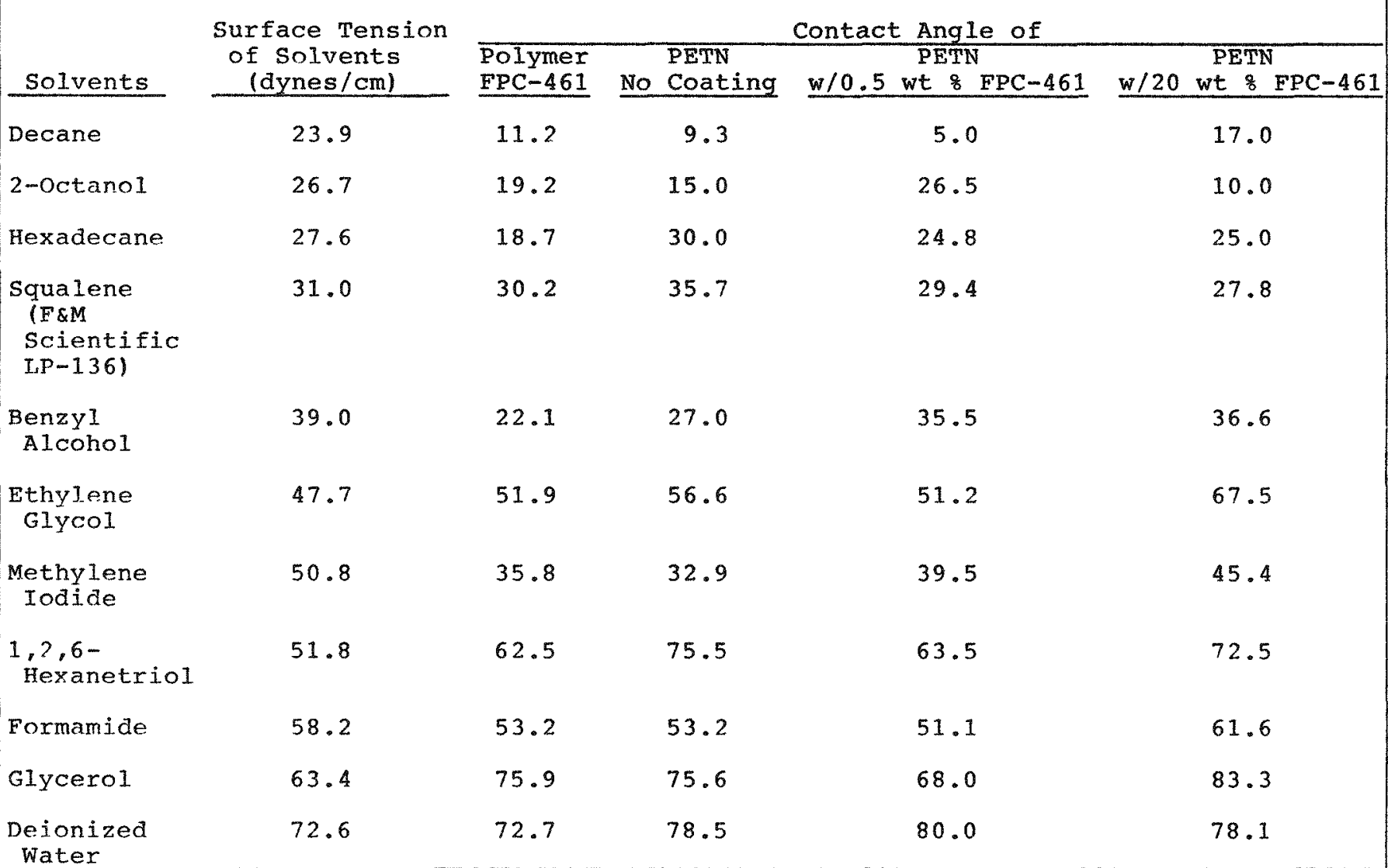

$x$-axis is the critical surface tension of that material.

Tables 3 and 4 show the contact angle measurement values obtained for smooth and rough pellets. Initially smooth pellets were pressed, as discussed in section 2, for experimentation. Later it was decided to press pellets with rough surfaces. The rough pellets were pressed at much lower pressures than were the smooth, which caused less compaction to occur; therefore, they retained more surface roughness. Table 5 gives roughness ratios for comparing rough to smooth pellets.

The data of Tables 3 and 4 indicate that solvents of high polarity such as ethylene glycol, 1,2,6-hexanetriol, and 

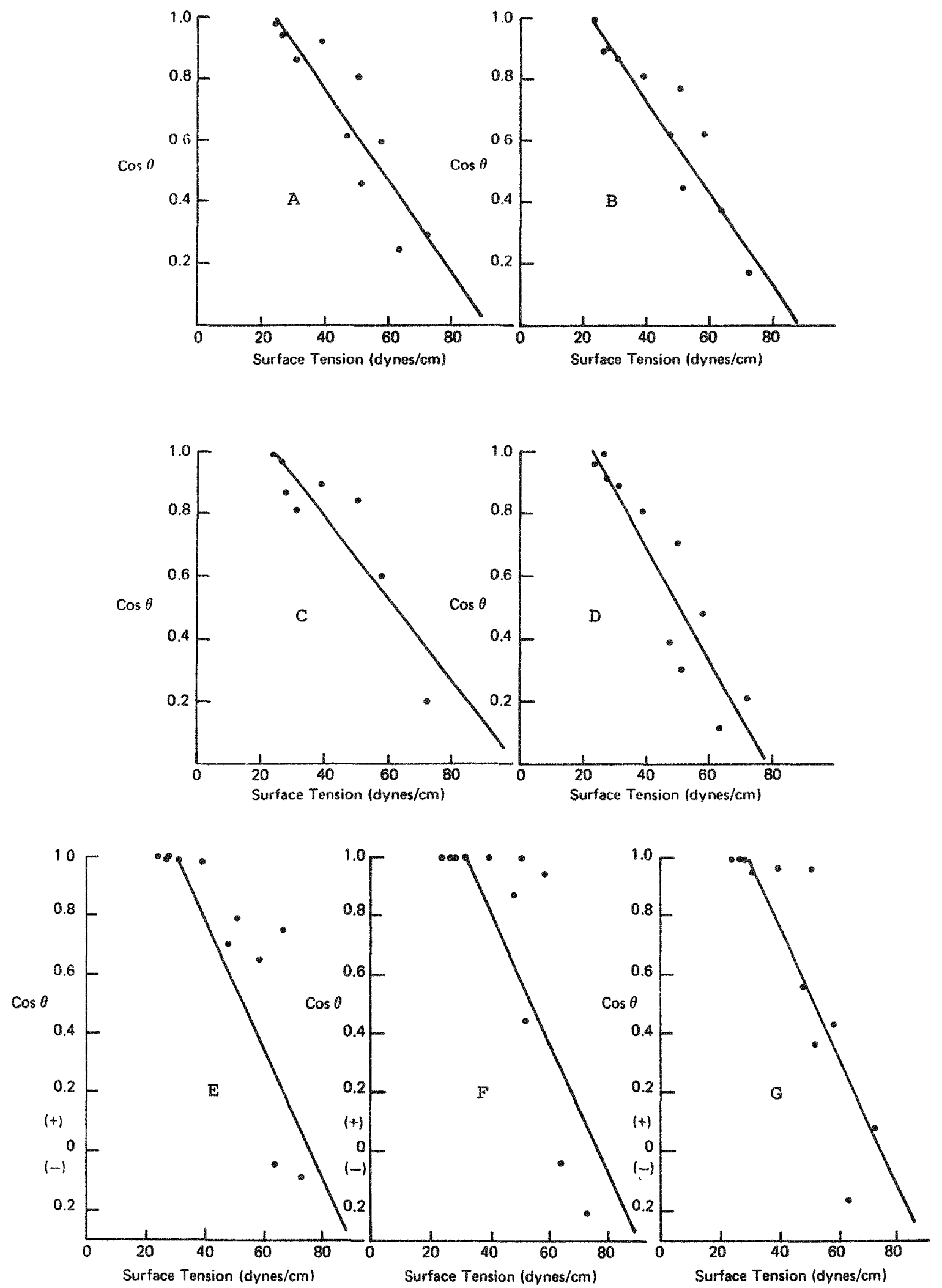

FIGURE 14 - Zisman plots of various solvents on (A) polymer-smooth pellets, (B) PETN pellet-no coating-smooth pellets, (C) 0.5 wt $\%$ coated PETN-smooth pellets, (D) 20 wt $\%$ coated PETN-smooth pellets, (E) polymer-rough pellets, (F) 0.5 wt \% coated PETN-rough pellets, and (G) 20 wt \% coated PETN-rough pellets. 
Table 4 - CONTACT ANGLE MEASUREMENTS OF VARIOUS SOLVENTS ON THE POLYMER AND THE UNCOATED SAMPLES OF PETN ER7479 (ROUGH PELLETS)

\begin{tabular}{|c|c|c|c|c|c|c|c|}
\hline \multirow[b]{3}{*}{ Solvents } & \multirow{3}{*}{$\begin{array}{c}\text { Surface Tension } \\
\text { of Solvents } \\
\text { (dynes/cm) }\end{array}$} & \multicolumn{6}{|c|}{ Contact Angle of } \\
\hline & & Polymer & PETN & & PETN & & PETN \\
\hline & & FPC-461 & No Coating & $w / 0.5$ & wt 8 FPC -461 & $w / 20$ & Wt $\&$ FPC -461 \\
\hline Decane & 23.9 & 0.0 & $-\infty$ & & 0.0 & & 0.0 \\
\hline 2-octannI & 26.7 & 8.5 & -- & & 0.0 & & 0.0 \\
\hline Hexadecane & 27.6 & 9.0 & -- & & 0.0 & & 5.0 \\
\hline $\begin{array}{l}\text { Squalene } \\
\text { (F\&M } \\
\text { Scientific } \\
\text { LP-136) }\end{array}$ & 31.0 & 9.1 & -- & & 0.0 & & 17.25 \\
\hline $\begin{array}{l}\text { Benzyl } \\
\text { Alcohol }\end{array}$ & 39.0 & 10.5 & $-\infty$ & & 0.0 & & 14.0 \\
\hline $\begin{array}{l}\text { Ethylene } \\
\text { Glycol }\end{array}$ & 47.7 & 45.3 & $-\infty$ & & 28.9 & & 55.6 \\
\hline $\begin{array}{l}\text { Methylene } \\
\text { Iodide }\end{array}$ & 50.8 & 37.5 & -- & & 0.0 & & 15.1 \\
\hline $\begin{array}{l}1,2,6- \\
\text { Hexanetriol }\end{array}$ & 51.8 & 55.6 & -- & & 63.25 & & 68.25 \\
\hline Formamide & 58.2 & 49.4 & -- & & 19.0 & & 64.2 \\
\hline GIycerol & 63.4 & 92.4 & -- & & 92.0 & & 99.2 \\
\hline $\begin{array}{l}\text { Deionized } \\
\text { water }\end{array}$ & 72.6 & 95.0 & -- & & 101.8 & & 85.2 \\
\hline
\end{tabular}

glycerol would be the least acceptable for use in coating PETN with FPC-461, because poor wettability would result. Figure 15 shows the discrepancy in the data from the polar solvents. The peaks from thrse polar solvents show an unexpected increase in the contact angle (decrease in $\cos \theta$ ) which results in a decrease in wettability.

The data of Table 3 and 4 also indicate that solvents, such as decane, that have Low contact angles with the FPC-46I and/ or PETN surfaces and low surface tensions would be ideal for the microencapsulation enhanced with starved addition process. It is essential that a solvent be used that will achieve wettability of the
FPC-461 on the PETN particles. Without good wettability properties, it is more difficult to achieve a coated powder.

The critical surface tension values determined from the zisman plots of Figure 14 (A-G) are shown in Table 6. These values indicate that a solvent surface tension less than or equal to these must be used to obtain sufficient wettability properties.

Methylene chloride (solvent used in cnating process) has a surface tension of 26.52 dynes/cm [5] which is close to decane and close to the critical surface tension values. Even though methylene chloride was not chosen as a solvent for 


\begin{tabular}{|c|c|c|c|c|c|}
\hline \multirow[b]{2}{*}{ Solvents } & \multirow{2}{*}{$\begin{array}{c}\text { Surface Tension } \\
\text { of Solvents } \\
\text { (dynes/cm) } \\
\end{array}$} & \multicolumn{4}{|c|}{ Contact Angle of } \\
\hline & & $\begin{array}{l}\text { Polymer } \\
\text { FPC-461 }\end{array}$ & $\begin{array}{l}\text { PETN } \\
\text { No Coating }\end{array}$ & $\begin{array}{c}\text { PETN } \\
\text { w/0.5 wt } 8 \text { FPC-461 }\end{array}$ & $\begin{array}{c}\text { PETN } \\
\text { W/20 wt } 8 \text { FPC-461 }\end{array}$ \\
\hline Decane & 23.9 & 1.019 & -- & 1.004 & 1.046 \\
\hline 2-octanol & 26.7 & 1.048 & -- & 1.117 & 1.015 \\
\hline Hexadecane & 27.6 & 1.056 & - & 1.101 & 1.099 \\
\hline $\begin{array}{l}\text { Squalene } \\
\text { (F\&M } \\
\text { Scientific } \\
\text { LP-136) }\end{array}$ & 31.0 & 1.142 & - & 1.148 & 1.079 \\
\hline $\begin{array}{l}\text { Benzyl } \\
\text { Alcohol }\end{array}$ & 39.0 & 1.061 & -- & 1.228 & 1.208 \\
\hline $\begin{array}{l}\text { Ethylene } \\
\text { Glycol }\end{array}$ & 47.7 & 1.139 & -- & 1.395 & 1.475 \\
\hline $\begin{array}{l}\text { Methylene } \\
\text { Iodide }\end{array}$ & 50.8 & 0.978 & - & 1.295 & 1.375 \\
\hline $\begin{array}{l}\text { 1,2,6- } \\
\text { Hexanetriol }\end{array}$ & 51.8 & 1.223 & -- & 1.009 & 1.229 \\
\hline Formamide & 58.2 & 1.087 & -- & 1.506 & 0.914 \\
\hline Glycerol & 63.4 & -0.172 & -- & -0.093 & -1.416 \\
\hline $\begin{array}{l}\text { Deionized } \\
\text { Water }\end{array}$ & 72.6 & -0.293 & -- & -1.172 & 0.408 \\
\hline
\end{tabular}

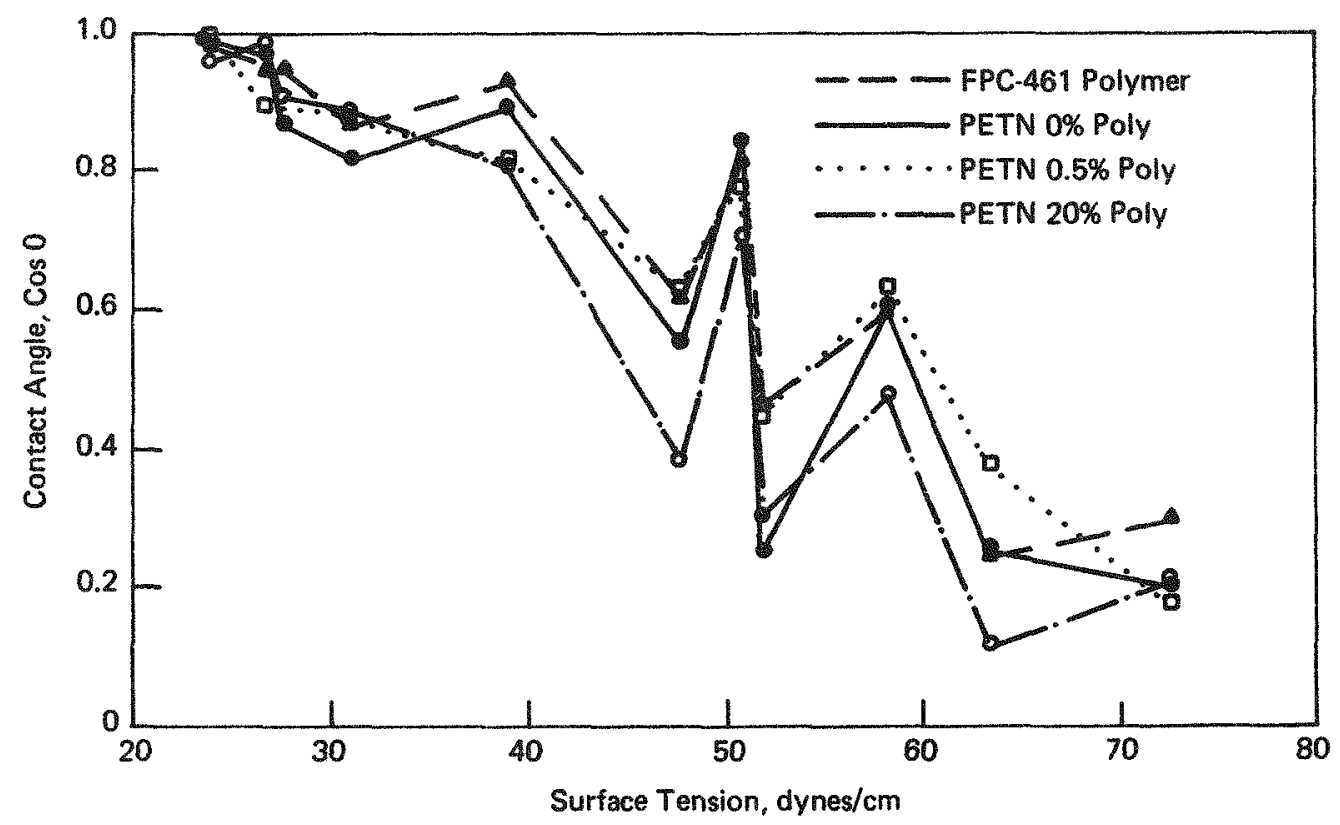

FIGURE 15 - Plot of contact angle measurements from various solvents on FPC-461 and coated and uncoated PETN pellets. 


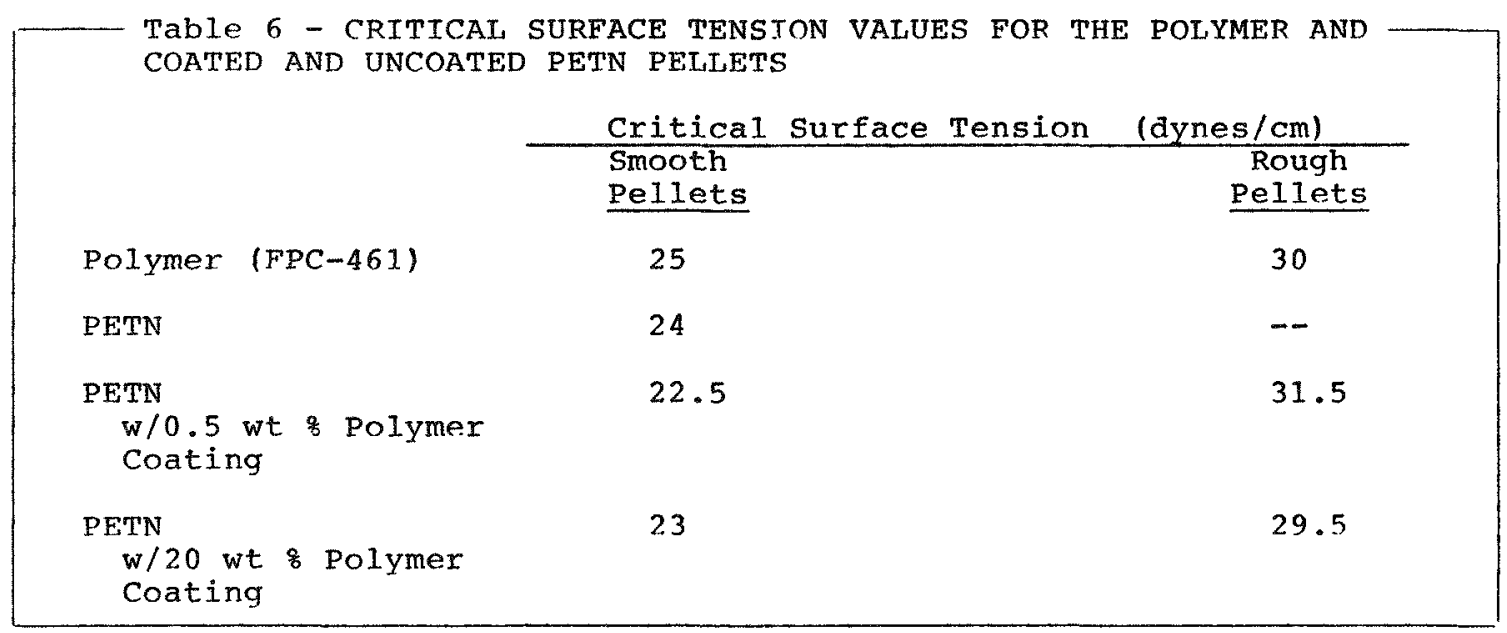

the contact angle measurement study, the results indicate that methylene chloride would be a good choice as a solvent in this application. A contact angle measurement of $10.2^{\circ}$ was obtained for methylene chloride on an uncoated PETN pellet, supporting the conclusion that methylene chloride would be a good solvent for the coating process.

\section{Summary}

Two models were proposed to aid in discussing the coating of an explosive powder using a microencapsulation technique enhanced with starved addition. Model I depicted a totally coated particle, whereas Model II depicted a partially coated particle. IsS results support the hypothesis of Model II for 0.5 wt 8 polymer.

Average thickness of the coating was calculated from $\mathrm{x}$-ray photoelectron spectroscopy to be $223 \AA$ and $1200 \AA$ for 0.5 and 20 wt $\&$ coated PETN, respectively. The average thickness was determined by coupling with ion scattering spectroscopy.

As discussed earlier in the text, Figure 1 (A\& B) illustrates the two proposed models. This figure also indicates that, for Model $I$, XPS because of its sampling depth $(20-40 \AA)$ would detect the FPC-461 and the PETN particle, whereas ISS would detect only the FPC-461 for Model $I$, since the sampling depth is much smaller than that of XPS. Because of the irregularities of the sample, XPS and ISS would detect both the FPC-461 and the PETN particle for Model II.

The results of XPS building energy values indicated that there was no change in the surface chemistry of the FPC-461 and/or the PETN after coating. This led to the belief that the type of bond occurring between PETN and FPC-461 was mechanical.

Contact angle measurements indicate that solvents of low surface tension are best for use in the microencapsulation enhanced by starved addition process. For best wettability, the work of Adhesion, $W_{S L}$ (See Appendix $\left.c\right)$, should be a small value. Results indicate that methylene chloride is a good choice for a solvent in the starved addition coating process.

Good depth and spatial resolution were obtained with the scanning electron microscope (SEM). The FPC-461 and PETN particles were easily viewed. The 
photodraphs showed evidence of the FPC461 on the 0.5 wt 8 coated PETN particles. The FPC-461 can be seen draping across the 20 wt 8 coated PETN particles in the $1500 x$ photograph.

optical microscopy did not show anything of significance other than the comparison of particle size.

SEM backscatter for chlorine analysis did not produce any useable result. This was due to problems with charging of the sample.

\section{References}

1. Vannet, Marcia D., and George I. Ball, "Utilization of Microencapsulation Coating Techniques to Achieve Priling and Enhance the Pourability of PETN Explosive Powder," MIM-MI-84-52-0003, Monsanto Research Corp. (1984), $15 \mathrm{pp}$.
2. Allen, T. "Particle size Measurement." 3 rd ed." Chap. 13, pp. 392-413, Chapman and Hall (1981).

3. Gibbs, T. R., and A. Popolato (ed.), "IASL Explosive Property Data," University of California Press (1980), p. 100 .

4. Dobratz, B. M." "LLNL Explosives Handbook: Properties of Chemical Explosives and Explosive Stimulants," UCRL-52997, Lawrence Livermore Laboratory (March 16. 1984), pp. 19-87.

5. Weast, R. C., "CRC Handbook of Chemistry and Physics," CRC Press, Inc. (1979), p. F-47. 


\section{Appendix A}

HISTORY OF MICROENCAPSULATION

The concept of microencapsulation was developed at the National Cash Register Company (NCR) in the late 1930s.[1] Barrett Green, a chemist at NCR, examined for several years the control of color formation in photographic applications. Green developed a product that would give multiple paper copies without the messy carbon black, duplication paper. The first gelatin microcapsules were prepared by Green in 1942 . These gelatin microcapsules were the result of Green's use of Bungenberg de Jong's coacervation concepts. $[2,3]$ Coacervation is the formation of a coating around a suspended particle. It took 9 yr to develop a marketable product, which was named NCR paper. The new product included a colorless dye-base in oil droplets in which a second sheet of paper was coated with an acidic clay (Figure A-1). The transfer of information occurs when pressure from the writing instrument fractures the microcapsules. [1] The colorless dye is encapsulated in the oil; and thus, when the capsule fractures, the dye reacts with the acidic clay to form the colored print seen on the second sheet.

Coacervation is divided into two types, simple and complex. [4] simple coacervation involves the presence of one colloid, whereas complex coacervation involves two or more colloids. Green used complex coacervation to prepare a colloid-rich gelatin coacervate phase into which droplets of oil were dispersed. $[5,6,7]$ The gelatin coacervate phase first wets and then coats the dispersed oil droplet, and the gelatin phase is hardened via a crosslinking agent.

Improvements in encapsulation efficiency were achieved by including a third colloid capable of carrying greater surface charges. These high-surface-charged colloids are added to the dispersion media, and they migrate to the dispersed particles. This, in turn, directly results in a higher degree of coacervation, and thus, microencapsulation. Improvements can also be made by carefully controling the drying process. slow drying produces microcapsules with low permeability. An example of this is that of low-boiling oils which can be held for extended periods without cracks forming in the coating.

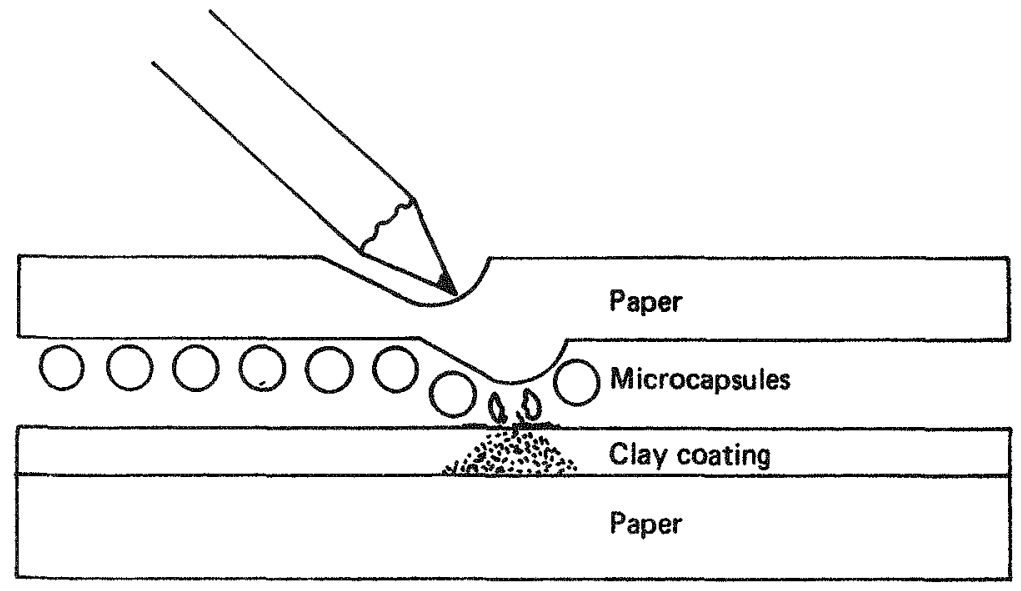

FIGURE A-1 - Illustration of duplication paper used in the market place.[1] 
Since it was not possible to encapsulate polar liquids directly in a gelatin colloid, an alternative procedure was perfected. In this process the interior oil core was exchanged with a polar liquid after the capsule wall had been prepared. [8] By swelling the microcapsule in water, the osmotic pressure present causes the exchange of the oil for a polar material. The result is a polar material coating a polar core material.

Once it was shown by NCR (1959) that aqueous solutions of gelatin could be utilized to contain organic solutions, a technique known as "phase separation" was perfected which allowed polar liquids to be encapsulated directly inside polymeric materials. At about the same time, Dr. Marco Cannalonga of Hoffman La Roche investigated various ways of protecting vitamins from premature decomposition. [9,10] New dehydration techniques were utilized to produce solid powders containing active vitamins with increased long-term stability.

As early as 1927. A. Bolk Roberts had spray dried emulsions of flavor oil. [11] In the early $1950^{\prime} \mathrm{s}$, technological advances led to the commercial drying and protection against long-term aqing of milk, coffee, and other food products. Spray drying requires heat to remove large volumes of water from the coating solution. In contrast to capsules prepared by coacervation and phase separation, spray dried capsules are not single droplet capsules, but instead are composed of hundreds of tiny dispersed oil droplets in a water-soluble polymer matrix.

At Battelle Memorial Institute, fluidized bed technology was tailored for microencapsulation. Solid-core particles are fluidized by a gas which keeps them suspended, usually in an inert bed of particles. Then the core particles are sprayed with a solution of coating material dissolved in a volatile solvent. This technique is especially applicable in coating pills, irregular shapes, and many edible products.112,13] The fluid bed technique is limited to capsules 200 um and 1 arger, because of particle agglomeration during coating.

A technique for microencapsulation which has not yet been commercialized is chemical vapor deposition. One example is the encapsulation of nuclear fuel particles, in which the core particles are coated with carbon by condensing thermally decomposed hydrocarbons on the suspended, moving core particles.[14] A lower capsule size limit of approximately $200 \mu \mathrm{m}$ can be expected with this technique.

In-situ polymerization was developed as a variation of chemical vapor deposition. The core particle can be either a solid or a liquid, and lower temperatures are generally employed. $[15,16]$

A nylon film was prepared by interfacial polymerization by dupont researchers in 1959.[17] The basic chemistry involved contacting an organic solution of acid chloride with an aqueous solution of a diamine to produce a film at the interface of two liquids. If droplets of one solution are added to the second, with stirring, microcapsules can be obtained. [4] 
In conclusion, a diverse and practical science has developed in the field of microencapsulation. Many methods of time-release and protective coatings have been devised to aid in our health and environment. There are also a number of uses for microencapsulation, such as carbonless paper, flavors and essences, pesticides and herbicides, pharmaceuticals, medical and veterinary applications, adhesives, and visual indicators.

Description of Microencapsulation

Microencapsulation is a process in which tiny particles or droplets are surrounded bv a coating (coacervate) to give small capsules (Figure A-2). In its simplest form, a microcapsule can be considered as a small sphere with a uniform wall around it. The material inside the microcapsule is referred to as the core, internal phase, or fill; the wall is sometimes called a shell, coating, or membrane. In this work, the inside material is referred to as the core particle and the wall material as the polymer coating. Most microcapsules have a diameter between a few micrometers to a few millimeters. [18]

Many microcapsules do not resemble simple spheres. The core may be a crystal, a notched adsorbent particle, an emulsion, a suspension of many particles, or even a suspension of microcapsules.

The reasons for microencapsulation include the isolation of the core particle from its surroundings to protect it from the deteriorating reactions with oxygen, to prevent evaporation of the core, to improve the handing properties of a sticky material, or to isolate a reactive core from chemical attack. In other cases, the objective is not to isolate the core but to control the rate at which it leaves the microcapsule. In the case here, a microencapsulation enhanced with starved addition technique was used to obtain a uniform distribution of polymer coating on the core particles. Uniform distribution, in this sense, means having some amount of polymer coating on all the core particles and not necessarily having each particle itself totally coated. A freeflowing powder can result by agglomerating these particles using the cnating as a glue. [19]

Process Parameters of Microencapsulation

There are many process parameters that can be adjusted to control the nature of the coating in a microcapsule product. The principal process parameters include composition of the solution, rates of coating addition, temperature of the media, and agitation

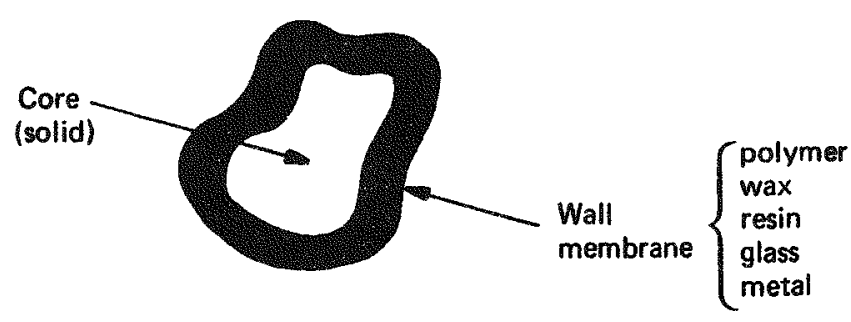

FIGURE A-2 - ITlustration of a microencapsulated particle.[18] 
speed which disperses the particles. In addition to these parameters, there are factors that affect the size and quality of microcapsules produced.

The diameter of the microcapsules depends upon the initial size of the core particle. The nature of the coating placed on core particles can be controlled by adjusting the agitation speed, the type and amount of dispersing agent (surfactant), the viscosity of the organic and aqueous phases, the configuration of the vessel and stirrer, the quantity of the organic and aqueous phases, and the temperature.[20]

The quality of the microcapsules is also influenced by several factors. Some of these factors include the rate of solvent removal, the molecular weight and crystallinity of the polymeric coating material, the type and concentration of the dispersing agent, choice of solvent or mixed solvent, the solubility of the core material in the continuous phase, achievement of low surface energies to ensure wettability, and the chemistry of the interface. Examples of solvents that may be used include water and water immiscible organics with boiling points less than $100^{\circ} \mathrm{C}$, such as aliphatic hydrocarbons, aromatic hydrocarbons, chlorinated hydrocarbons, and Freons. various cosolvents such as alcohols, ketones, esters, and plasticizers may also be used as solvents. Examples of dispersing agents that may be used include polymeric materials such as polyvinyl alcohol, polyvinyl pyrxolidone, carboxymethyl cellulose, gelatin, and starch. Nonionic, cationic, and anionic dispersing agents can also be used, as well as solid dispersing agents such as talc, magnesium sulfate, and calcium carbonate.

The best polymeric materials to use for coating are those with low surface tensions. Usually polymers with molecular weights of at least 10,000 work best. In general, amorphous, rather than crystalline polymers, make good coatings for microcapsules prepared by solvent evaporation [21] because they are less ordered and, therefore, more soluble in a solvent.

Before a microencapsulation technique can be chosen for a particular application, there are physical properties that must be carefully considered, such as core wettability, core solubility, coating permeability, and coating elasticity.

In coacervation coating, the critical property is the wettability of the core by the coacervate. If properly wet, solid particles are easier to coat than liquid cores. proper wetting may be difficult if a liquid core material is highly insoluble in the coacervate-forming solution. [18]

The wettability of a solid of a particular coacervation system is easily determined, but it is rare to have a particle surface of proper configuration available for accurate measurement of the spreading coefficient; a measurement of wettability. The wettability can be monitored during the microencapsulation process by observing the ability of the coacervate particles to coat the core particles sufficiently. 
It is critical that the core not be soluble in the polymer solvent and that the polymer not be soluble in the dispersing medium.

The determination of the solubility of the reactants in the phases permits a choice of solvents and polymers.

The polymer permeability indicates whether a core can be isolated. The microcapsules must be able to tolerate handling but may be required to break at a predetermined threshold pressure. The coating polymer, core size, and coating thickness determine elasticity and friability. [18]

Concentration and temperature are other important variables of concern. The coating polymer can become sticky or tacky and cause clumping over certain concentration and temperature ranges. Melting point, glass-transition temperature, degree of crystalInity, coating-degradation rate, and many other properties have to be considered.

As discussed earlier, there are many parameters that control the degree of microencapsulation.[20] To examine all would be impossible in this work.

The main factors studied in this work were the wettability and chemistry of the interface. The chemistry of the interface involves the nature of the bonding, its thickness, and the distribution of the coating. This chemistry was determined by the surface analysis techniques that are discussed in Appendix $C$. The wettability studies were done to determine surface tension compatibility of the polymer, energetic materials, and adhesive for the final product. Surface tension results were discussed in section 3 . 


\section{Appendix B}

APPLICATION OF A COATING BY A STARVED ADDITION ENHANCED MTCROENCAPSULATION TECHNIOUE The microencapsulation technique chosen for this project was solvent evaporation, enhanced by starved addition. [19] The general scheme is shown in Figure B-1. The procedure for this technique involves the following steps. First, an emulsion is made. An emulsion is a mixture of mutually insoluble liquids in which one is dispersed in droplets throughout the other (e.g., oil in water). The microdroplets that form, a discontinuous phase, are a solution consisting of a polymeric coating material, a core particle to be encapsulated, and an organic solvent. The water involved is called the processing medium or the continuous phase. Once the emulsion has stabilized, the solvent is gradually removed from the microdroplets by evaporation. The solvent partitions out of the microdroplets into the continuous phase as shown in $F$ igure B-2. As the solvent is removed, microcapsules take the shape of the particle being coated as is illustrated in Figure B-3. When all solution has been removed, the particles no longer stick together as easily. The result is particles that are easier to hande.

The solvent evaporation technique used in this work varied from the process described above. The core particles were suspended in a water-ethanol mixture. The polymeric coating (FPC-461) was introduced drop-by-arop by starved addition to the dispersed media of PETN. PETN is the core material consisting of needle-shaped particles 30 um in diameter. Starved addition is defined as very slow addition of the polymer-solvent solution to the dispersed media of PETN. Once the solution was stable, the solvent was gradually removed from the microdroplets by evaporation; particles, each coated with a small amount of polymer, resulted. This process is illustrated in Figure B-4.

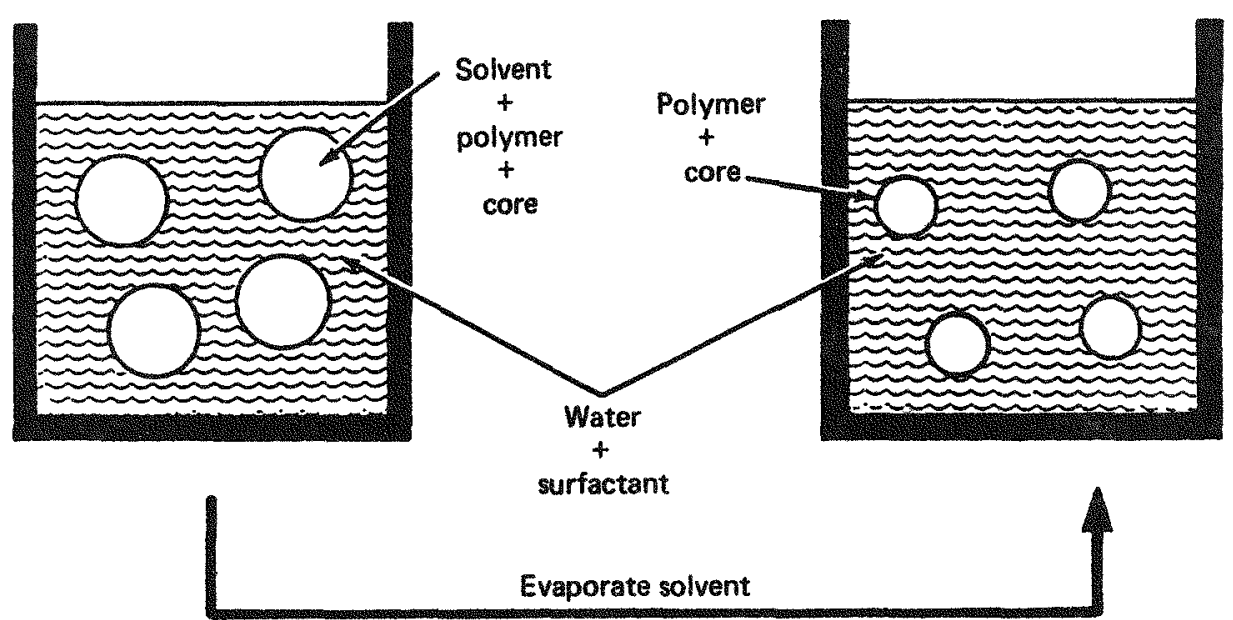

FIGURE B-1 - The general scheme of microencapsulation by the solvent evaporation process. [25] 


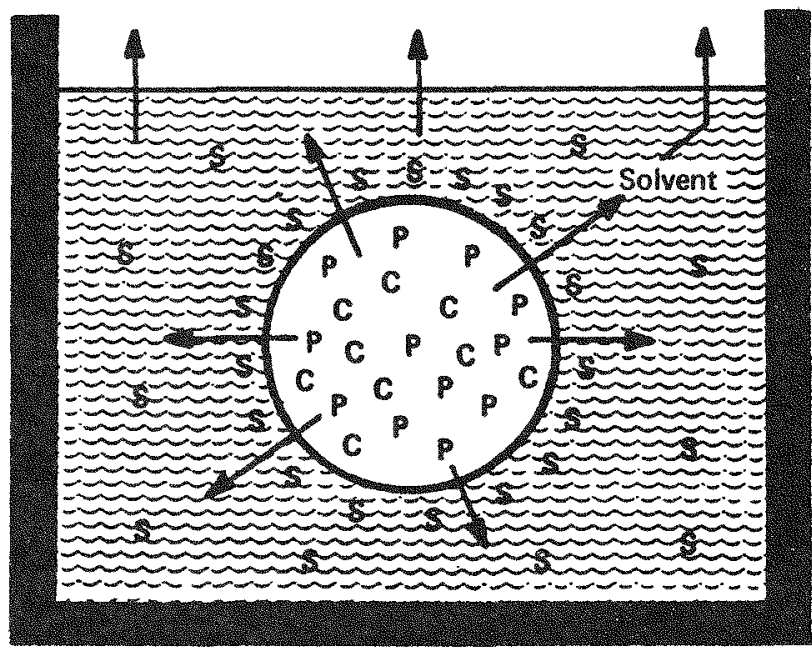

$\left.\begin{array}{l}P=\text { polvmer } \\ C=\text { core }\end{array}\right]$ in soivent

FIGURE B-2 - Illustration of organic solvent partitioning from microdroplets first into the aqueous phase and then evaporation occurs.[21]

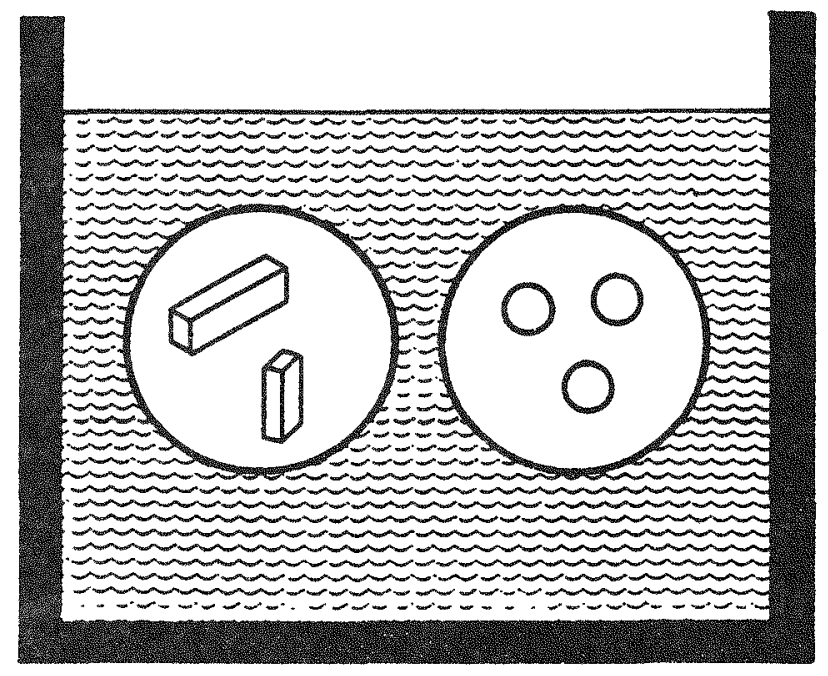

FIGURE B-3 - Illustration of the various shapes and sizes of microcapsules that can form. [21] 

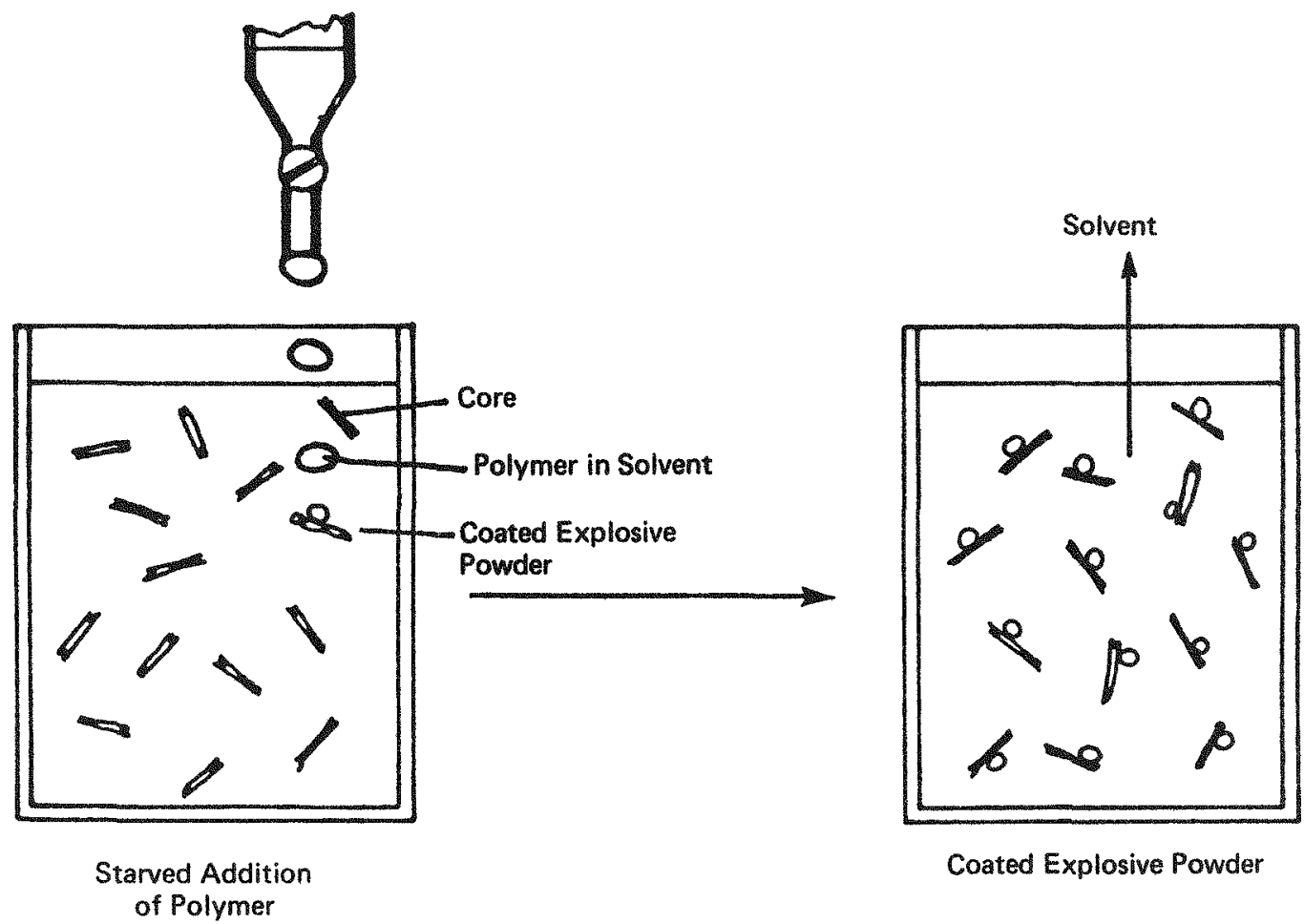

FIGURE B-4 - Illustration of the solvent evaporation coating technique utilizing starved addition of the polymer.[19] 


\section{Appendix C}

SURFACE ANALYTICAL TECHNIQUES

Optical Microscopy

Optical microscopy has been a popular means of magnifying objects. The microscope is designed to resolve features in order to permit two closely spaced objects to be viewed separately. The optical microscope is used to examine particles from about 0.8 to $150 \mu \mathrm{m}$ in diameter. Above $150 \mu \mathrm{m}$, a microscope is useful, but for smaller particles it is necessary to use a secondary electron microscope. [22] The most severe limitation of the optical microscope is its small depth of focus. The edges of the images seen in a microscope are blurred as a result of diffraction effects. The conventional optical microscope can resolve features down to the wavelength of visible light, or about $5000 \AA$.

Scanning Electron Microscopy

The scanning electron microscope (SEM) is a widely used instrument for examining surfaces. Spatial resolution to within a few hundred angstroms is possible depending on the nature of the sample. Figure $c-1$ is a schematic of the sEM indicating how the surface is scanned by a focused electron beam, and how the intensity of secondary electrons is monitored. The output from the secondary electron detector modulates the raster of a cathode-ray tube, which is scanned in synchronization with the focused electron beam. Fach point on the cathode-ray tube (CRT) raster corresponds to a point on the surface of the sample, and the strength of the image at each point varies according to the intensity of secondary electron production from the corresponding point on the surface. The image quality depends on having a high signal intensity so that a wide variation in signal is possible and, therefore, good contrast in the image. sem images characteristically have a wide range of contrast in which detail can be seen both in very dark and in very light areas. The images also have great depth of focus; they are sharp at both very low and very high points of the surface. [23]

Figure $\mathrm{C}-2$ illustrates the electron scattering that occurs on the coated PETN surface in the SEM. The electron beam incident on the sample surface causes various phenomena, of which the emission of secondary electrons is the most commonly used. The emitted electrons strike the collector, and the resulting current is amplified and used to modulate the brightness of a CRT. The times associated with the emission and collection of the secondary electrons are negligibly small compared with the times associated with the scanning of the incident electron beam across the sample surface. Therefore, there is a one-to-one correspondence between the number of secondary electrons collected from any particular point on the sample surface and the brightness of the analogous point on the CRT screen. ConsequentIy, an image of the surface is progressively built up on the screen. $[24]$

\section{SEM Backscatter}

Some of the high-energy incident electrons undergo Rutherford scattering from the sample atoms and reemerge from the surface. The resulting image is in some ways like the 


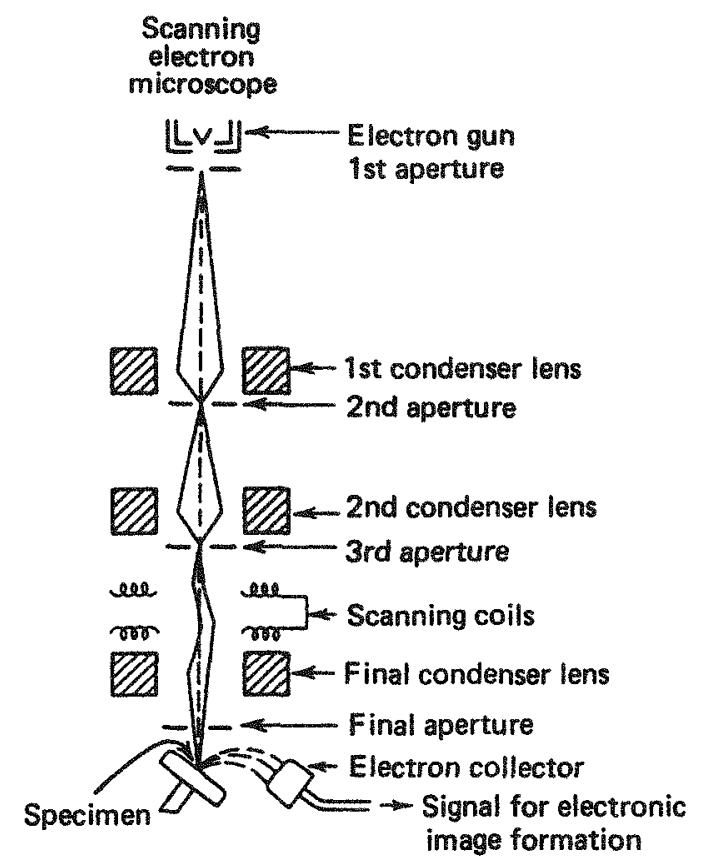

FIGURE $C-1$ - Schematic of image formation in the scanning electron microscope. [23]

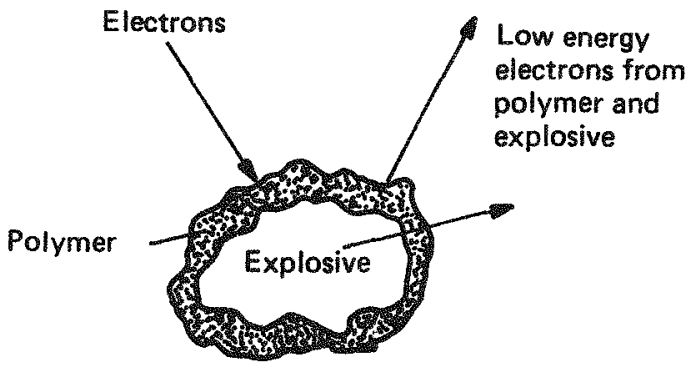

FIGURE C-2 - Electron scattering on a coated and/or uncoated PETN particle in the SEM.

secondary electron image, but there are a number of differences. First, the backscattered electrons come from a greater depth in the sample, and because of the spreading of the electrons in the sample, they represent a larger area, and thus give poorer resolution, rarely better than $200 \mathrm{~nm}(2000$ A). [25] Since the backscattered electrons come from deeper in the sample, they contain less information about the surface and more about the bulk material. Also, since they are of greater energy, they are not affected significantly by applied voltages or valence bonding in the material.

The backscattered electron signal is monotonically dependent on the specimen"s average atomic number (Z), but with the fraction of electrons backscattered increasing as $z$ increases. [25] Higher-z elements have more electrons and hence give rise to a greater number of backscattered electrons and a smaller number of absorbed electrons than lower- $z$ elements do. This result assumes that all other factors contributing to contrast are equivalent from the low- and high-z regions. Ideally this would be the 
case when the surface is flat and uniformly conducting so that geometrical and charging contributions, to the atomic-number contrast are reduced to practically zero. However, this mode of information is usable only for relatively large atomic-number differences. [24] The atomic number difference between the chlorine of FPC-461 and carbon of the PETN material was nine. It was hoped that the backscatter coefficients would be large enough that the presence of the coating could be identified.

To use the SEM in characterizing surfaces, coating of the sample is essential. Particle samples of the energetic materials were carbon coated to prevent charging of the sample in the SEM system. The carbon coating grounds the sample and also affixes the sample so the electron beam will not throw the particles off the mounting stub.

$x$-ray Photoelectron Spectroscopy

$\mathrm{X}$-ray photoelectron spectroscopy (XPS) is a technique used to determine the electronic structure of solid surfaces as well as surface components.

In an XPS experiment $x$-ray photons, hv, from a monoenergetic beam hit a sample surface. The photons are absorbed by the sample atoms, resulting in the emission of an electron. Electrons from all the orbitals of the atom with a binding energy, $E_{b}$, less than the $x$-ray energy are excited. Since all of the atomic orbitals are not excited with equal probability, some peaks are more intense than others in the spectra. Energy is conserved; therefore the kinetic energy, $\mathrm{KE}$, of the electron, plus the energy required to remove it from its orbital to the spectrometer vacuum, must equal the $x$-ray energy. Using the known $x$-ray energy and measuring the kinetic energy with the electron spectrometer, one can obtain the binding energy in the atomic orbital. During the calibration procedure, a spectrometer work function, $\phi$, must be applied for solid state experiments. Thus, one obtains $E_{b}=h v-K E+\emptyset_{s} \cdot[26]$

The basic elements of an $\mathrm{x}$-ray photoelectron spectrometer are shown in Figure $\mathrm{C}-3$. The functions of the spectrometer are to produce intense $x$-radiation, to irradiate the sample to photoeject core electrons, to introduce the ejected electrons into an energy analyzer, to detect the energy-analyzed electrons, and to provide a suitable output of signal intensity as a function of electron binding energy. There are a number of commercial instruments available which accomplish these functions, each using a different approach for design of the source, energy analyzer, and detector. [26]

Electron spectroscopy is sensitive to all elements but hydrogen and helium. The atomic structure of each element in the periodic table is aistinct from all the others, therefore measurement of the positions of one or more of the electron lines allows quick identification of an element present at a sample surface. Each of the elements in PETN produces at least one electron line in a spectrum. These electron lines are well separated from one another so that no discrepancy exists in identification of adjacent elements. For the PETN material, one would expect to see photoelectron lines from 


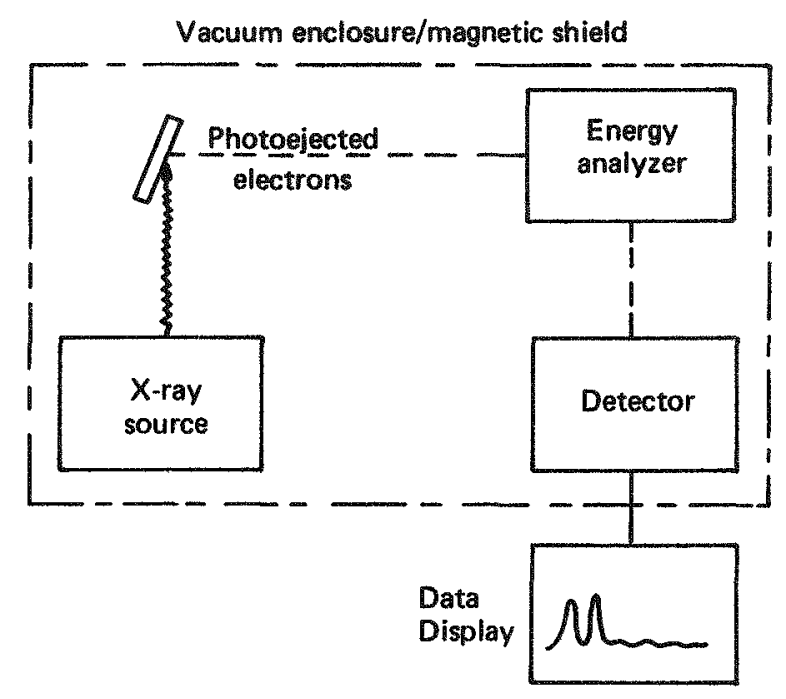

FIGURE C-3 - Basic elements of an x-ray photoelectron spectrometer. [26]

carbon, nitrogen, and oxygen. From FPC-461, one would expect to see photoelectron ines from carbon, fluorine, and chlorine. Thus, FPC-461 and PETN are easily distinguished from one another.

Quantitative information can be obtained as well as qualitative. The intensity of the electron signals is proportional to the number of similar atoms in the sample. [27] Observation of the signal intensities can provide semiquantitative and quantitative analyses. The XPS signal intensities depend upon the mean free path, MFP, of the electrons and the efficiency of absorption of the exciting $x-r a y s$ in the sample material. The MFP is on the order of $20-40 \AA$ in polymers.

Although the $x$-rays penetrate deep into the sample, the photoejected electrons emerge from within only a few molecular layers of the surface. The MFp of an electron is proportional to the exponential power of the kinetic energy. The chemical properties deduced from XPS data are those of surface molecules.

A quantity also measured in XPS is the binding energy ( $E_{b}$ ) of the ejected electron. Chemical shift is the observed changes in the binding energies of core levels resulting from changes in chemical bonding. It is observed that removing valence electron density from an atom increases the observed binding energy of a core level. [28]

A core electron is subject to a combination of forces, the resultant of which is known as its binding energy. From the nucleus, there is a strong attractive force proportional to the magnitude of the nuclear charge or atomic number. The outer, or valence-shell, electrons exert a repulsive force which screens the core electron from the nuclear charge, diminishing the nuclear attractive force. $E_{b}$ is the binding energy by which the electron is bound to the atom. 
If an electron is removed from the outer shell, the screening of the inner electrons is reduced by one electron charge, and the core electrons therefore have an increased force of attraction from the nucleus. A negative change in oxidation state, i.e., the gain of an outer electron, has the opposite effect, effectively increasing the shielding and decreasing $E_{b}$.

The binding energy of the nitrogen in the explosive, PETN, would be found at 408.2 ev. This would be characteristic of a nitrogen in a highly oxidized state, such as in the RONO 2 structure of an explosive. The binding energy of the chlorine and fluorine of the polymer would be characteristic of the chlorines and fluorines bound in an organic polymer, such as FPC-461.

A meaningful determination of a chemical shift depends upon measuring the absolute binding energy of an atomic level. It is not possible with a nonconducting sample simply to use the observed binding energy values because the surface may become charged [28] and retard the photoejected electrons. This decrease in the kinetic energy of the photoelectrons results in higher apparent binding energies than the true values [29] and limits the amount of chemical information obtainable from the data. The mean potential at which the surface charge stabilizes depends on the electron emission from the sample, the photoejected electrons from the $x$-ray window and sample chamber walls that impinge on the sample, and the electron conduction in the sample surface. [301 The magnitude of the charge effect will vary from instrument to instrument.

A technique for charge neutralization is to flood the sample environment with low-energy electrons causing a negative surface charge, which accelerates the photoelectrons rather than retarding them. The peak observed on a charged surface may be broadened because of the variation in the charge across the sample surface, or because of a charge gradient on the surface. The rationale for using charge neutralization is that a minimum line width corresponds to zero surface charge; it assumes that the observed species is in electrical equilibrium with the sample and accurately tracks the charge on the sample. In the absence of electrical equilibrium, the binding energies measured for other surface species will be in error. [26]

Ion Scattering spectroscopy

Ion scattering spectroscopy (ISS) is a technique used to analyze the elements present on the outermost atomjc monolayer of a surface. The surface is bombarded with a beam of noble or inert gas ions $\left({ }^{3} \mathrm{He}^{+},{ }^{4} \mathrm{He}^{+},{ }^{20} \mathrm{Ne}^{+}\right.$, or $\left.{ }^{40} \mathrm{Ar}^{+}\right)$. A fraction of these ions ( 1 in 10 ${ }^{5}$ ) undergoes a single binary elastic collision with a surface atom. This interaction, which takes place in roughly $10^{-16} \mathrm{~s}$, changes the energy and momentum of these primary ions and scatters them. It is these scattered ions that are analyzed and that contajn the information revealing the elemental composition of the outermost atomic monolayer of the surface. The energy change (energy loss) is related to the mass of the scattering center (atom) on the surface by: 


$$
\frac{E_{1}}{E_{0}}=\frac{M_{0}^{2}}{\left(M_{0}+M_{S}\right)^{2}}\left[\left(2 \cos \theta+\frac{M_{S}{ }^{2}}{M_{0}^{2}}-\sin \theta\right)^{1 / 2}\right]^{2}
$$

where $M_{0}=$ mass of the analyzing gas ion:

$\mathrm{M}_{\mathrm{s}}=$ mass of atom on the sample surface;

$\mathrm{E}_{0}=$ kinetic energy of $\mathrm{M}_{0}$ before collision;

$E_{1}=$ kinetic energy of $M_{0}$ after collision; and

$\theta=$ scattering angle for $M_{0} \cdot[31]$

A monoenergetic, collimated beam of ions strikes the target surface (sample), and the energy distribution of ions scattered off at an angle of $138^{\circ}$ is measured. Figure C-4 is a schematic of the ISS apparatus. The result is an energy spectrum which provides information on the mass, the chemical identity, and the number of atoms on the surface. These peaks are given by a combination of ion and target atoms which occur at fixed values of $E_{1} / E_{0} \cdot[32]$ It would be expected that the ISS signals for carbon, nitrogen, oxygen, fluorine, and chlorine from the PETN explosive and the FPC-461 polymer would be observed at $E_{1} / E_{0}$ values of $0.410,0.468,0.516,0.574$, and 0.745 with a scattering angle of $138^{\circ}$.

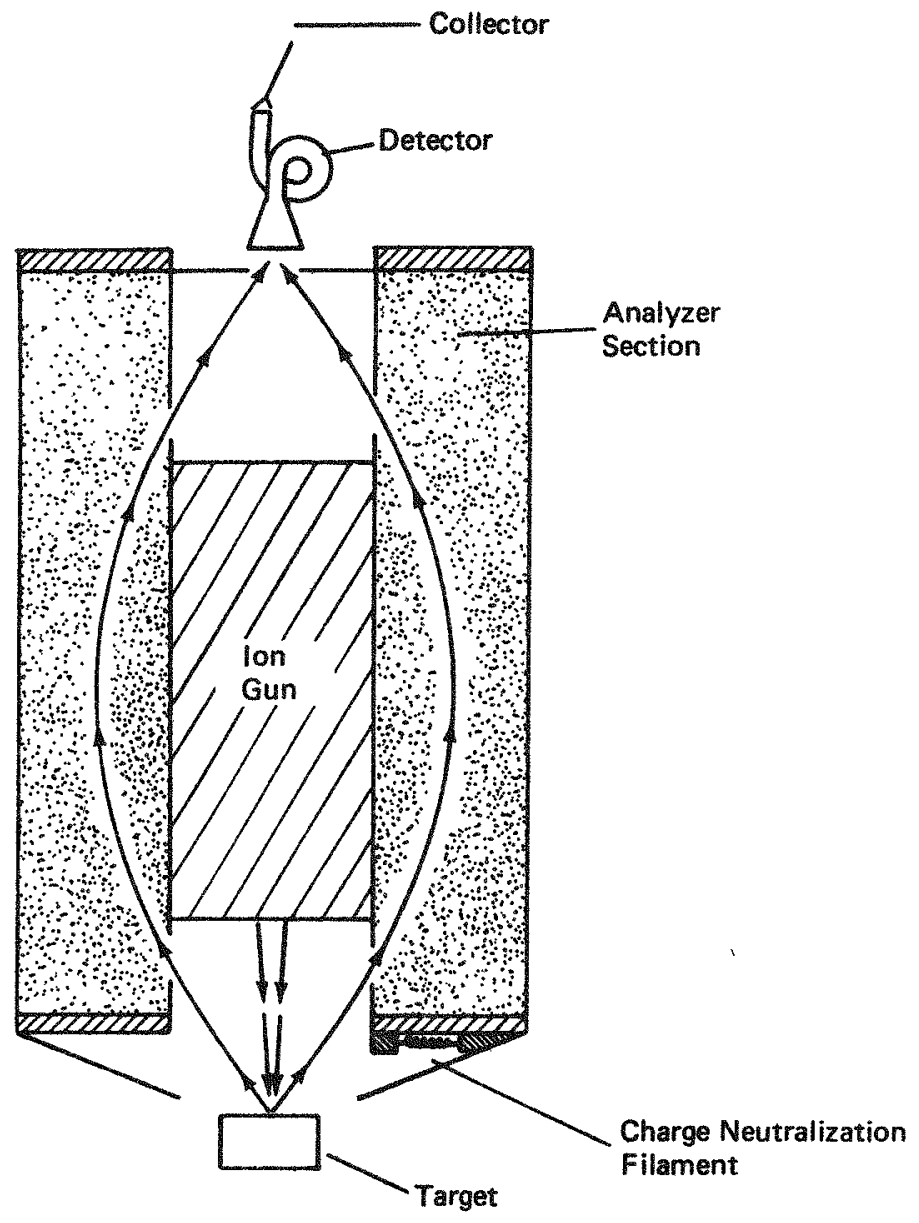

FIGURE C-4 - Schematic of the ISS apparatus. 
The experiment therefore involves probing the surface with a primary beam in the energy range of several kev $(0.5$ to 2 is sufficient) and energy analyzing the reflected primary beam. If the primary beam (ion) penetrates the surface beyond the first atomic layer, it has a low probability of undergoing a simple binary collision inside the bulk material and then scattering back out without losing any additional energy. Therefore, this technique is very surface sensitive, and one expects the signal which is obtained to be related completely to the surface monolayer with a very limited contribution from the second or third atom layers.

Depth profile analysis, with possible monolayer resolution, is extremely useful in studies involving surface composition gradients, studies relating surface to bulk properties, studies of film thickness and composition as a function of thickness, and studies of contamination and penetration into the sample. This unique ability to examine a surface, layer by layer, is particularly important on most technological surfaces where the greatest compositional changes occur in approximately the first 20 monolayers. [31]

Sputtering rates vary with the analyzing gas, and depend on the nature of the sample. For helium ions the sputtering rate generally ranges from 3 to 50 monolayers/hr. With the relatively slow removal rate $(3$ monolayers/hr) it is possible to examine carefully a - single monolayer. When higher sputtering rates are desired, more massive noble gas ions, either neon or argon, are used.[31] Removal rates are approximately 5 to 10 times greater, respectively.

All types of surfaces, from insulator to metallic, can be analyzed with the ISs technique. The only condition that must be attained is that the material must have a low vapor pressure, in order for the system to maintain a vacuum. Nonconductive surfaces are examined with the aid of the charge neutralization system. Neutralization eliminates the surface charge buildup by bathing the sample with thermally emitted electrons, while the sample remains at a zero potential. Insulating surfaces can be examined directly with neutralization. The charge neutralization system operates via feedback stabjilization between the electron source and the total measured target current. [31]

Particle Size (Coulter Counter) - Surface Area

The coulter technique is a method of determining the number and size of particles suspended in an electrolyte by causing them to pass through a small orifice on either side of which is immersed an electrode as shown in Figure $c-5$. The changes in resistance as particles pass through the orifice generate voltage pulses whose amplitudes are proportional to the volumes of the particles. The pulses are amplified, sized, and counted; from the derived data the size distribution of the suspended phase may be determined. [33]

The particle size analysis obtained showed the needle-shaped PETN uncoated powder sample to be $9.8 \mathrm{\mu m}$ in diameter (at $50 \%$ cumulative volume). The coated sample could not be 


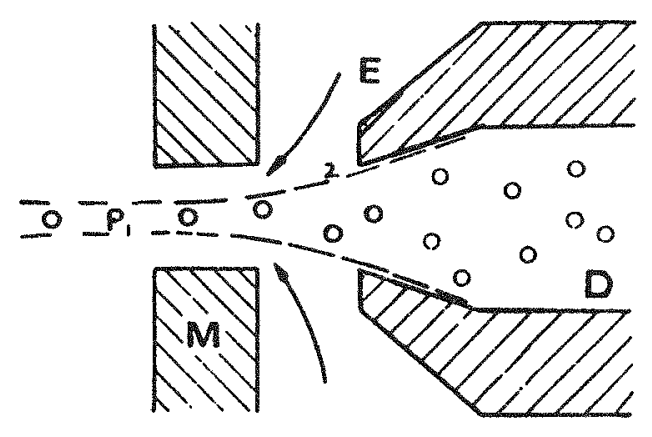

FIGURE C-5 - Schematic of suspended particles passing through an orifice in which electrodes monitor changes in resistance. [33]

tested because of the desired particle agglomeration which was introduced by the presence of FPC-461. This caused the individual particles to adhere to one another. The size distributions are difficult to characterize because of the irregular shape of the particles.

Although the Coulter Counter technique does not give an accurate measurement of the particle size of the irregular (needle like) particles of PETN, the Coulter Counter data may be used to give an estimated value for the surface area of the PETN particles.

Surface area can be calculated using the following equations:

$$
\begin{aligned}
& V=4 / 3 \pi r^{3} \\
& A=4 \pi x^{2} \\
& D=M / V \\
& S=A / M=3 / D \times x \text { per } g
\end{aligned}
$$

$$
\text { where } \begin{aligned}
\mathrm{V} & =\text { volume, } \mathrm{cm}^{3} \\
\mathrm{M} & =\text { mass, } \mathrm{g} \\
\mathrm{A} & =\text { area }, \mathrm{cm}^{2} \\
\mathrm{D} & =\text { density, } \mathrm{g} / \mathrm{cm}^{3} \\
\mathrm{~S} & =\text { specific surface area, } \mathrm{cm}^{2} / \mathrm{g} \\
\mathrm{r} & =\text { average radius, } \mu \mathrm{m}
\end{aligned}
$$

The density for PETN is $1.78 \mathrm{~g} / \mathrm{cm}^{3}$.

Calculation of specific surface area from coulter counter data resulted in a value of $3440 \mathrm{~cm}^{2} / \mathrm{g}$ for the uncoated sample of PETN. This value is far below the value of 12,600 $\mathrm{cm}^{2} / \mathrm{g}$ as determined by the Fisher subsieve method for uncoated PETN. This discrepancy 
in the surface area value is due to the calculation assumption in the coulter Counter measurements that the particles are spherical, where in reality each particle is very irregular (needle like) in shape.

Contact Angle

There are various methods of measuring the contact angle of a liquid on a solid. The method most commonly used, and the one chosen for this project, is that of measuring $\theta$ directly for a drop of liquid resting on a flat surface of the solid. This measurement is made with a goniometer telemicroscope (Figure $\mathrm{C}-6$ ). This drop of liquid (or solvent) is placed under the action of three surface tensions (j), which are depicted in Figure C-7: ${ }_{\mathrm{LV}}$ at the interface of the Iiquid and vapor phases, ${ }^{2}$ SI at the interface of the solid and vapor. If the equilibrium tensions are resolved horizontally, Young's equation is obtained.

$$
{ }_{S V}=\partial_{S L}+\partial_{L V} \cos \theta
$$

An equivalent equation, as stated by Dupre, is known as the definition of work of adhesion,

$$
\mathrm{W}_{\mathrm{SI}}=\partial_{\mathrm{IV}}(1+\cos \theta)
$$

This derivation assumes that the vertical force from ${ }_{\mathrm{LV}}$ produces no deformation of the solid surface. [34,35]

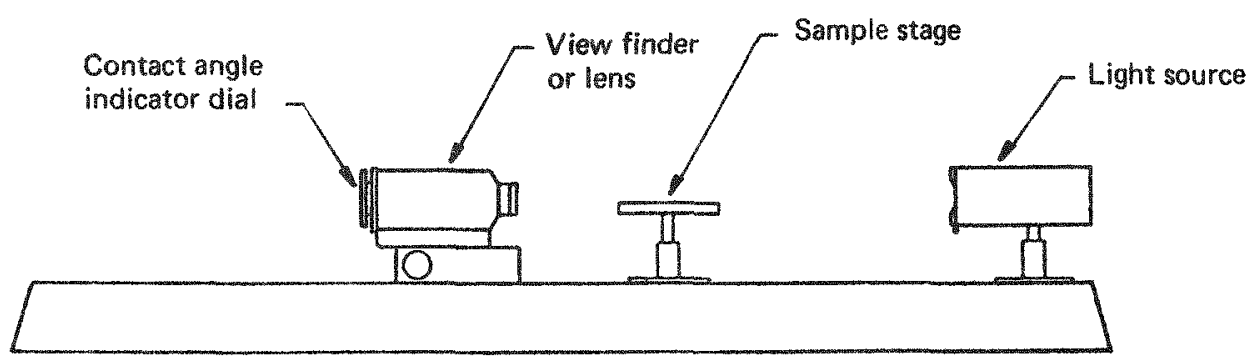

FIGURE C-6 - Schematic of goniometer telemicroscope.

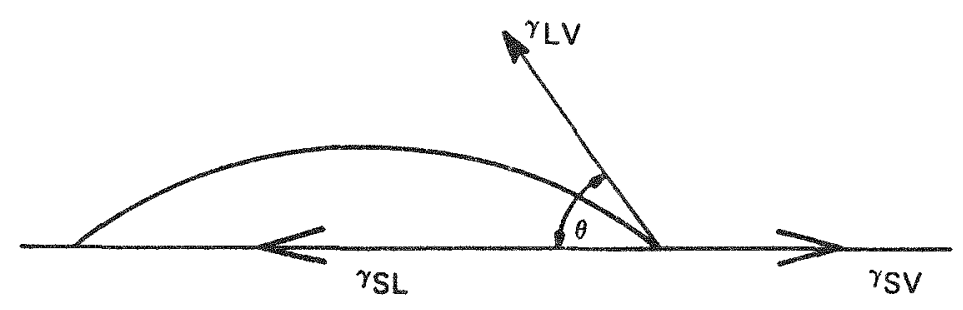

FIGURE C-7 - IlTustration of surface tensions placed on drop of 1iquid (solvent). r351 
Young's equation (Eq. 6) indicates that the magnitude of the contact angle will depend on the relative values of the adhesion between solid and Iiquid and on the mutual cohesion of the 1 iquid which is related to ${ }^{2} \mathrm{LV}^{\circ}$ An angle of $180^{\circ}$ would indicate zexo adhesion, but this angle has never been observed in practice, even with hydrophobic materials and water. The contact angle is also dependent on the roughness of the surface and on any contamination of the solid surface or of the liquid surface, or possible rearrangement of the solid surface caused by the presence of the liquid phase. $[34,35]$

Surface roughness has the effect of making the contact angle further from $90^{\circ}$. If the smooth, solid gives a contact angle greater than $90^{\circ}$, the angle is increased by roughening the surface (tendency to spread increases); if the contact angle is less than $90^{\circ}$, then roughness will decrease it. Wenzel has proposed that surface roughness may be measured by:

$$
R=\cos \theta^{\prime} / \cos \theta
$$

where $R$ is the roughness ratio, $\theta^{\prime}$ is the average contact angle on a rough surface, and $\theta$ is the average contact angle on a smooth surface. This relationship is only applicable to submicroscopic roughness, since for coarse roughness the edge becomes ragged and $R$ is no longer a constant. This relationship is important because surfaces having $R=1.00$ are rarely encountered. Most of the time, $R$ is much greater than one. [34,35] Wenzel's equation was derived (Eq. 8) from the Young equation which assumes that the contact angle is independent of the volume of the drop and depends only on the temperature and the nature of the liquid, solid, and vapor phases in contact.

References

1. Fanger, G. O. in "Microencapsulation: Processes and Applications," J. E. Vandegaer (ed.), Plenum Press, New York (1974) pp. 1-20.

2. Bungenburg de Jong, H. G., Proc. Acad. Sci. Amsterdam 41, 646 (1938).

3. Bungenburg de Jong, H. G., Kass, Biochem. Zeit., 232, 338, (1931).

4. Gutcho, M. H., "Microcapsules and Microencapsulation Techniques," Noyes Data Corporation (1976), p. 33 .

5. Green, B. K., U.S. Reissue 24,899 (November 29, 1960).

6. Green, B. K., and L. Schleicher, U.S. 2,800,457 and U.S. 2,800,458 (July 23, 1957).

7. Bakan, J. A., U.S. 3,436,355 (Apri1 1, 1969).

8. Brynko, C., and G. M. Olderman, U.S. 3,516,943 (June 23, 1970). 
9. Taylor, H. F., U.S. 2,183,053(1939) and U.S. 2,218,592 (1940).

10. Koff, A., and P. F. Widmer, U.S. 3,143,475 (January 23, 1961 ).

11. Grevenstuk, A. B., and F. Hougesteger, U.S. 3,202,731 (August 24, 1965).

12. Sachse1, G. F,, and H. Nack, U.S. 3,202,533 (August 24, 1965).

13. Nack, H., U.S. 3,035,338 (May 29, 1962) and U.S. 3,382,093 (May 7, 1968).

14. Powe11, C. F., J. H. Oxley, and J. M. Blocker, Ir. (ed.), "Vapor Deposition," John Wiley \& Sons (1966).

15. Herman, D. F., U. Kruse, J. J. Brancato, "Bolyethylene Encapsulated Cellulose," J. Poly. Sci. Part C. No. 11, 75-95 (1965).

16. Herman, D. F., and U. Kruse, U.S. 3,297,466 (January 10, 1967 ).

17. Biston, J. H., "Permeability of plastic, The Flavor Industry," (November 1970), pp. $779-783$.

18. Flinn, J. E., and H. Nack, "What Is Happening In Microencapsulation," Chemical Engineering, (December 4, 1967), pp. 171-178.

19. Vannet, M. D., and G. L. BaII, "Utilization of Microencapsulation Coating Techniques To Achieve Prilling and Enhance The Pourability of PETN Explosive Powder," MLM-ML-84-52-0003, Monsanto Research Corporation, (1984), p. 15.

20. Moodie, W. J., and J. E. Bennett, "Batch and Continuous Recrystallization of Secondary Explosives," MLM-3137, Monsanto Research Corporation, (March 9, 1984 ).

21. Sparks, R. E. - Course Director, The Center for Professional Advancement Microencapsulation (Seminar) - Section G - "Microencapsulation Using Solvent Evaporation" P.O. Box H, East Brunswick, New Jersey 08816, Dr. Thomas R. Tice, June $25-27,1984$.

22. Allen, T., "Particle size Measurement," 3rd ed. Chapman and Ha11 (1981), p. 187.

23. Adamson, A. W., "Physical Chemistry of surfaces, 4th ed. John Willey \& Sons (1982), pp. 284-299.

24. Jahari, 0. and A. V. Samudra in "Characterization of Solid Surfaces," P. F. Kane and G. B. Larrabee (ed.), Chap. 5, Plenum Press, New York (1974), pp. 107-131. 
25. Russ, J. C., in "Systematic Materials Analysis," J. H. Richardson and R. V. Peterson, (ed.), Volume II, Chap. 16, pp. 159-181, Academic Press, Inc.. New York (1974).

26. Riggs, W. M., and M. J. Parker in "Methods of Surface Analysis," A. W. Czanderna (ed.), Volume 1 of Methods and Phenomena: Their Applications in Science and Technology, Chap. 4, pp. 103-158, Elsevier Scientific Publishing Co. (1975).

2.7. Hercules, S. H., and D. M. Hercules in "Characterization of Solid Surfaces," P. F. Kane and G. B. Larrabee, Chap. 13, pp. 207-336, Plenum Press, New York (1974).

28. Proctor, W. G. in "Systematic Materials Analysis," J. H. Peterson and R. V. Peterson (ed.), Volume II, Chap. 19, pp. 229-251, Academic Press, Inc., New York (1974).

29. Danis, W. P., J. E. Lester, Anal. Chem., 45 (1973) 1416.

30. EbeI, M. F., H. Ebel, J. Electron Spectrosc., 3 (1974) 169.

31. Carbonara, R. S., in J. H. Richarason and R. V. Peterson, (ed.), "Systematic Materials Analysis," Volume III, Chap. 25, pp. 229-240, Academic Press, Inc. $(1974)$.

32. Buck, T. M., in A. W. Czanderna, (ed.), "Methods of Surface Analysis," Volume I of Methods and Phenomena: Their Applications in Science and Technology, Chap. 3, pp. 75-102, Elsevier Scientific Publishing Co. (1975).

33. Allen, T., "Particle size Measurement," 3rd ed., Chap. 13, pp. 392-413, Chapman and HaII (1981).

34. Maycock, M. J., in "Dispersion of Powders," G. D. Parfitt (ed.), pp. 22-30, Elsevier Publishing Company Limited (1969).

35. Gould, R. F. (ed.), "Contact Angle, Wettability, and Adhesion," Advances in Chemistry Series 43, pp. 1-56, American Chemical Society, Washington, D.C. (1964). 


\section{Distribution}

\section{EXTERNAL}

TIC, UC-4 (145)

J.A. Morley, DOF/DAO

R. George, DOE/AL

D. Plywale, DOE/AI

$\mathrm{V}$. Aschembrener, DOE/AL

\section{Los Alamos National Laboratory}
C. B. Banks
R. K. Rohwer
H. H. Cady
A. P. Torres
E. M. Hanson
D. G. Tuggle
W. F. Hemsing
W. W. Whaley
T. F. Larson
R. J. Yactor

\section{Sandia National Iabnratories, Albuquerque}

D. H. Anderson

J. G. Harlan

J. E. Kennedy

T. M. Massis

\section{Pantex}

R. Slape

H. W. Merritt

R. Bailey

G. Osborne

D. E. Mitche11

R. H. VanDomelen

P. D. Wilcox
Sandia National Laboratories, Livermore
D. I. I.inaner
I. A. West

Lawrence Livermore National Laboratory
M. Finger
D. I. Seaton
H. A. Golopol
R. R. McGuire
R. C. Ragaini
W. H. Siegel
N. A. Schneider
D. Wright

R. W. Whan

\section{General Electric Neutron Devices}
A. J. Mione
R. Walton

\section{Unidynamics/Phoenix}

C. T. Rittenhouse

INTERNAL
A. Attalla
P. S. Back
G. I. Ball (5)
B. D. Barton
R. A. Benson
J. D. Braun
J. R. Brinkman
D. A. Buckner
A. R. Burke
R. S. Carlson
J. C. Cheng
D. L. Coffey
I. A. $\operatorname{Cox}$
R. J. DeSando (3)
J. V. Dichairo
T. A. Donaldson
I. R. Dosser
W. R. Feairheller
C. L. Fellers
T. K. Ferquson
D. W. Firsich
R. A. Fischbein
J. R. Gibson

\author{
J. E. Glaub \\ F. G. Ha1I \\ J. I. . Harrison \\ I. D. Haws \\ E. D. Hill \\ D. I. Hobrock \\ W. B. Hogeman \\ D. Holman \\ J. A. Holy \\ T. W. Hughes \\ C. W. Huntington \\ P. L. Johnson \\ D. Kapsch \\ D. P. Kelly \\ M. D. Kelly \\ B. R. Kokenge \\ J. M. Lavoie \\ J. W. Laffingwell \\ F. D. Lonadier \\ C. M. Love \\ G. D. Miller \\ W. E. Moddeman \\ J. H. Mohler
}
A. C. Munger
G. L. Numbers
W. Pardieck
K. D. Phipps
J. M. Pickard
J. W. Reed
R. F. Salerno
D. R. Schaeffer
D. R. Spangler
J. A. Thomes
R. Thorpe
P. Trainer
R. E. Vallee
M. D. Vannet
P. S. Wang
D. E. Wendeln
R. B. Whitaker
C. J. Wiedenheft
C. M. Woods
C. M. Worley (3)
J. A. Zink
Publications
Document Control

Marjorie F. Hauenstein, Editor 